\title{
Higher order fluctuations and correlations of conserved charges from lattice QCD
}

\author{
Szabolcs Borsanyi, ${ }^{a}$ Zoltan Fodor, ${ }^{a, b, c}$ Jana N. Guenther, ${ }^{a, d}$ Sandor K. Katz, ${ }^{d}$ \\ Attila Pasztor, ${ }^{a}$ Israel Portillo, ${ }^{e}$ Claudia Ratti $^{e}$ and K.K. Szabó ${ }^{a, c}$ \\ ${ }^{a}$ Department of Physics, University of Wuppertal, \\ Wuppertal D-4209\%, Germany \\ ${ }^{b}$ Eötvös University, \\ Budapest 1117, Hungary \\ ${ }^{c}$ Jülich Supercomputing Centre, \\ Jülich D-52425, Germany \\ ${ }^{d}$ Department of Physics, University of Regensburg, \\ Regensburg D-93053, Germany \\ e Department of Physics, University of Houston, \\ Houston, TX 7r204, U.S.A. \\ E-mail: borsanyi@uni-wuppertal.de, fodor@bodri.elte.hu, \\ Jana.Guenther@t-online.de, katz@bodri.elte.hu, \\ apasztor@bodri.elte.hu, iportillovazquez@gmail.com, cratti@uh.edu, \\ szaboka@general.elte.hu
}

ABSTRACT: We calculate several diagonal and non-diagonal fluctuations of conserved charges in a system of $2+1+1$ quark flavors with physical masses, on a lattice with size $48^{3} \times 12$. Higher order fluctuations at $\mu_{B}=0$ are obtained as derivatives of the lower order ones, simulated at imaginary chemical potential. From these correlations and fluctuations we construct ratios of net-baryon number cumulants as functions of temperature and chemical potential, which satisfy the experimental conditions of strangeness neutrality and proton/baryon ratio. Our results qualitatively explain the behavior of the measured cumulant ratios by the STAR collaboration.

KeYwords: Lattice QCD, Phase Diagram of QCD, Quark-Gluon Plasma

ARXIV EPRINT: 1805.04445 


\section{Contents}

1 Introduction 1

2 Fluctuations and imaginary chemical potentials 2

3 Analysis details $\quad 4$

3.1 Lattice setup 4

3.2 Expected result for $\chi_{8}^{B} \quad 5$

$\begin{array}{lll}3.3 & \text { Correlated fit with priors } & 6\end{array}$

3.4 Cross-correlators 8

$\begin{array}{ll}3.5 & \text { Error analysis }\end{array}$

4 Phenomenology at finite chemical potential 11

5 Conclusions and outlook $\quad 14$

A Results for the correlators $\quad 15$

B Statistics and lattice details $\quad 19$

C Data tables 20

\section{Introduction}

One of the most challenging goals in the study of Quantum Chromodynamics (QCD) is a precise mapping of the phase diagram of strongly interacting matter. First principle, lattice QCD simulations predict that the transition from hadrons to deconfined quarks and gluons is a smooth crossover [1-6], taking place in the temperature range $T \simeq 145-165 \mathrm{MeV}$. Lattice simulations cannot presently be performed at finite density due to the sign problem, thus leading to the fact that the QCD phase diagram is still vastly unexplored when the asymmetry between matter and antimatter becomes large.

With the advent of the second Beam Energy Scan (BES-II) at the Relativistic Heavy Ion Collider (RHIC), scheduled for 2019-2020, there is a renewed interest in the heavy ion community towards the phases of QCD at moderate-to-large densities. A rich theoretical effort is being developed in support of the experimental program; several observables are being calculated, in order to constrain the existence and location of the QCD critical point and to observe it experimentally.

Fluctuations of conserved charges (electric charge $Q$, baryon number $B$ and strangeness $S$ ) are among the most relevant observables for the finite-density program for several reasons. One possible way to extend lattice results to finite density is to perform Taylor expansions of the thermodynamic observables around chemical potential $\mu_{B}=0$ [7-11]. Fluctuations of conserved charges are directly related to the Taylor expansion coefficients of such observables, thus, they are needed to extend first principle approaches to the regions of the phase diagram relevant to RHIC. An other popular method to extend observables 
to finite density is the analytical continuation from imaginary chemical potentials [12-16]. The agreement between the analytical continuation and Taylor expansion was shown for the transition temperature with physical quark masses by Bonati et al. in ref. [17].

Fluctuations can also be measured directly, and a comparison between theoretical and experimental results was used to extract the chemical freeze-out temperature $T_{f}$ and chemical potential $\mu_{B f}$ as functions of the collision energy [18-22]. Such fluctuations have been recently calculated and extrapolated using the Taylor method in ref. [23]. Finally, higher order fluctuations of conserved charges are proportional to powers of the correlation length and are expected to diverge at the critical point, thus providing an important signature for its experimental detection [9, 24, 25].

In this paper, we calculate several diagonal and non-diagonal fluctuations of conserved charges up to sixth-order and give estimates for higher orders, in the temperature range $135 \mathrm{MeV} \leq T \leq 220 \mathrm{MeV}$, for a system of $2+1+1$ dynamical quarks with physical masses and lattice size $48^{3} \times 12$. We simulate the lower-order fluctuations at imaginary chemical potential and extract the higher order fluctuations as derivatives of the lower order ones at $\mu_{B}=0$. This method has been successfully used in the past and proved to lead to a more precise determination of the higher order fluctuations, compared to their direct calculation [26, 27]. The direct method (see e.g. [7]) requires the evaluation of several terms and is affected by a signal-to-noise ratio which is decreasing as a power law of the spatial volume $V$, with an exponent that grows with the order of the susceptibility.

We also construct combinations of these diagonal and non-diagonal fluctuations in order to study the ratio of the cumulants of the net-baryon number distribution as functions of temperature and chemical potential by means of their Taylor expansion in powers of $\mu_{B} / T$. We discuss their qualitative comparison with the experimental results from the STAR collaboration, as well as the validity of the truncation of the Taylor series.

The paper is organized as follows: we first discuss the use of imaginary chemical potentials in section 2. Section 3 gives details on the lattice setup, on the fitting procedure, on its generalization for cross-correlators, and finally on the error estimation. The phenomenological results for the ratios of kurtosis, skewness and variance of the baryon number are presented in section 4. Conclusions and outlook are discussed in section 5, while in the appendix we present all diagonal and non-diagonal fluctuations needed to construct the cumulant ratios shown in section 4 , and give additional technical details.

\section{Fluctuations and imaginary chemical potentials}

The chemical potentials are implemented on a flavor-by-flavor basis, their relation to the phenomenological baryon $(B)$, electric charge $(Q)$ and strangeness $(S)$ chemical potentials are given by

$$
\begin{aligned}
\mu_{u} & =\frac{1}{3} \mu_{B}+\frac{2}{3} \mu_{Q} \\
\mu_{d} & =\frac{1}{3} \mu_{B}-\frac{1}{3} \mu_{Q} \\
\mu_{s} & =\frac{1}{3} \mu_{B}-\frac{1}{3} \mu_{Q}-\mu_{S} .
\end{aligned}
$$


The observables we are looking at are the derivatives of the free energy with respect to the chemical potentials. Since the free energy is proportional to the pressure, we can write:

$$
\chi_{i, j, k}^{B, Q, S}=\frac{\partial^{i+j+k}\left(p / T^{4}\right)}{\left(\partial \hat{\mu}_{B}\right)^{i}\left(\partial \hat{\mu}_{Q}\right)^{j}\left(\partial \hat{\mu}_{S}\right)^{k}},
$$

with

$$
\hat{\mu}_{i}=\frac{\mu_{i}}{T}
$$

These are the generalized fluctuations we calculated around $\mu=0$ in our previous work [28].

The fermion determinant $\operatorname{det} M(\mu)$ is complex for real chemical potentials, prohibiting the use of traditional simulation algorithms. For imaginary $\mu$, however, the determinant stays real. The chemical potential is introduced through weighted temporal links in the staggered formalism:

$$
U_{0}(\mu)=e^{\mu} U_{0}, \quad U_{0}^{\dagger}(\mu)=e^{-\mu} U_{0}^{\dagger}
$$

Thus, an imaginary $\mu$ translates into a phase factor for the antiperiodic boundary condition in the Dirac operator. Due to the $Z(3)$ symmetry of the gauge sector, there is a non-trivial periodicity in the imaginary quark chemical potential $\mu_{q} \rightarrow \mu_{q}+i(2 \pi / 3) T$, which translates to the baryochemical potential as $\mu_{B} \rightarrow \mu_{B}+i 2 \pi T$, the Roberge-Weiss symmetry. This is independent of the charge conjugation symmetry $\mu_{B} \leftrightarrow-\mu_{B}$. As a result, e.g. for the imaginary part of the baryon density:

$$
\left.\langle B\rangle\right|_{\mu_{B} / T=i \pi-\epsilon}=-\left.\langle B\rangle\right|_{\mu_{B} / T=i \pi+\epsilon}
$$

At $\mu_{B}=i \pi T$ there is a first order phase transition at all temperatures above the Roberge-Weiss critical end point $T_{R W}$ [29]. When $\mu_{B}$ crosses $i \pi T$ in the imaginary direction, the imaginary baryon density is discontinuous. This behaviour is illustrated in figure 1, where the imaginary baryon density as a function of the imaginary chemical potential is shown. At low temperature the Hadron Resonance Gas model predicts $\langle B\rangle \sim \sinh \left(\mu_{B} / T\right)$, thus for imaginary values we expect a sine function below $T_{c}: \operatorname{Im}\langle B\rangle \sim \sin \left(\operatorname{Im} \mu_{B} / T\right)$. At temperatures slightly above $T_{c}$, we observe that further Fourier components appear in addition to $\sin \left(\operatorname{Im} \mu_{B} / T\right)$ with alternating coefficients, these are consistent with a repulsive interaction between baryons [30]. At very high temperatures, on the other hand, $\langle B\rangle$ is a polynomial of $\mu_{B}$ since the diagrams contributing to its $\sim \mu_{B}^{5}$ and higher order components are suppressed by asymptotic freedom [31,32]. The Stefan-Boltzmann limit is nonvanishing only for two Taylor coefficients of $\operatorname{Im}\langle B\rangle$, giving $\left.\operatorname{Im}\langle B\rangle\right|_{\mu_{B} / T=i \pi-\epsilon}=8 \pi / 27$. At finite temperatures above $T_{R W}$ this expectation value is smaller but positive. By eq. (2.5), it implies a first order transition at $\mu_{B}=i \pi T$.

The order of the transition at $T_{R W}$ heavily depends on the quark masses [33, 34]. For physical quark masses one obtains $T_{R W}=208(5) \mathrm{MeV}$, and the scaling around the endpoint is consistent with the Ising exponents [35]. This implies that, for physical parameters, the transition is limited to $\mu_{B}=i \pi T$ without any other structures between the imaginary interval $[0, i \pi)[33]$. 


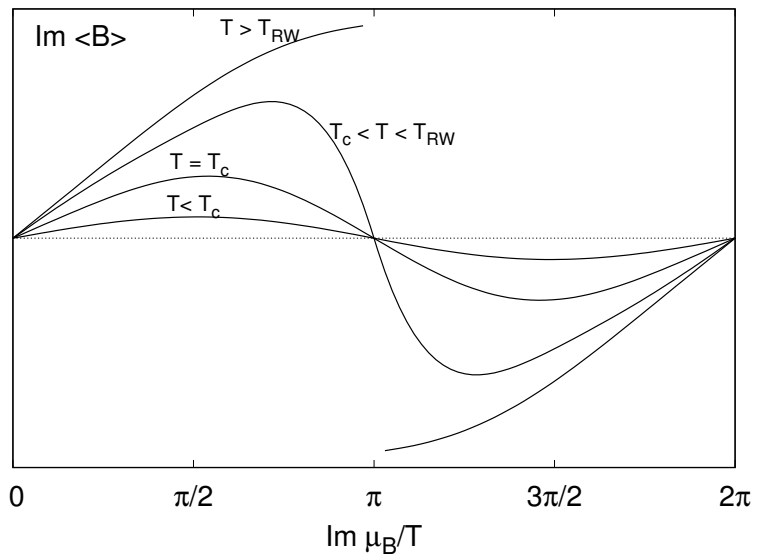

Figure 1. Cartoon for the imaginary baryon number $\left(\operatorname{Im} \chi_{1}^{B}\right)$ as a function of the imaginary chemical potential. $T_{R W}$ is the temperature of the Roberge-Weiss critical point.

Thus, we have only the range $\mu / T \in[0, i \pi)$ to explore the $\mu$-dependence of the observables. Recent simulations in this range include the determination of the transition line, where the slope was determined on the negative side of the $T-\mu_{B}^{2}$ phase diagram. Using analyticity arguments, this coefficient gives the curvature of the transition line on the real $T-\mu_{B}$ phase diagram [36-38]. Apart from the transition temperature, we used imaginary chemical potentials also to extrapolate the equation of state to real $\mu_{B}[26]$, which serves as an alternative approach to the Taylor extrapolation [39]. In an recent study D'Elia et al. have used the low order fluctuations at imaginary chemical potentials to calculate generalized quark number susceptibilities [27].

\section{Analysis details}

\subsection{Lattice setup}

In this work we calculate high order fluctuations by studying the imaginary chemical potential dependence of various generalized quark number susceptibilities.

We use a tree-level Symanzik improved gauge action, with four times stout smeared ( $\rho=0.125)$ staggered fermions. We simulate $2+1+1$ dynamical quarks, where the light flavors are tuned in a way to reproduce the physical pion and kaon masses and we set $\frac{m_{c}}{m_{s}}=11.85$ [40]. For the zero-temperature runs that we used for the determination of the bare masses and the coupling, the volumes satisfy $L m_{\pi}>4$. The scale is determined via $f_{\pi}$. More details on the scale setting and lattice setup can be found in [28].

Our lattice ensembles are generated at eighteen temperatures in the temperature range $135 \ldots 220 \mathrm{MeV}$. We simulate at eight different values of imaginary $\mu_{B}$ given as: $\mu_{B}^{(j)}=i T \frac{j \pi}{8}$ for $j \in\{0,1,2,3,4,5,6,7\}$. In this work the analysis is done purely on a $48^{3} \times 12$ lattice, we leave the continuum extrapolation for future work.

In terms of quark chemical potentials we generate ensembles with $\mu_{u}=\mu_{d}=\mu_{s}=\mu_{B} / 3$. In each simulation point we calculate all derivatives in eq. (2.2) up to fourth order. Thanks to our scan in $\operatorname{Im} \hat{\mu}_{B}$, we can calculate additional $\mu_{B}$ derivatives. Ref. [27] uses various 
"trajectories" in the $\mu_{B}-\mu_{Q}-\mu_{S}$ space, allowing the numerical determination of higher e.g. $\mu_{Q}$ and $\mu_{S}$ derivatives. We find relatively good signal for the $\mu_{Q}$ and $\mu_{S}$ derivatives by directly evaluating eq. (2.2) within one simulation. We recently summarized the details of the direct calculation in ref. [28].

\section{$3.2 \quad$ Expected result for $\chi_{8}^{B}$}

Before we embark into the discussion of the fit procedure we outline our expectations for the higher order fluctuations. It is an established fact that $T_{c}$ changes with the chemical potential $[17,36-38]$ resulting in a positive curvature $\kappa$ of the phase diagram with:

$$
T_{c}=T\left(1-\kappa \frac{\mu_{B}^{2}}{T^{2}}+\mathcal{O}\left(\frac{\mu_{B}^{4}}{T^{4}}\right)\right)
$$

Let us make a simple observation regarding the observed pattern of the baryon densities at imaginary chemical potential. At leading order in $\hat{\mu}_{B}$ the baryon density is simply $\chi_{1}^{B}\left(T, \hat{\mu}_{B}\right) \approx \chi_{2}^{B}(T, 0) \hat{\mu}_{B}$. Thus, at very small chemical potential $\chi_{1}^{B}\left(T, \hat{\mu}_{B}\right) / \hat{\mu}_{B} \approx$ $\chi_{2}^{B}(T, 0)$. In the left panel of figure 2 we show this ratio in the limit of $\mu_{B}=0$, that is, we plot $\chi_{2}^{B}(T, 0)$ and interpolate with a spline. We have access to the same ratio $\chi_{1}^{B}\left(T, \hat{\mu}_{B}\right) / \hat{\mu}_{B}$ at larger imaginary chemical potentials as well, one example $\left(\hat{\mu}_{B}=i 5 \pi / 8\right)$ is plotted with blue dots in figure 2. This curve is essentially similar, but its inflection point has been shifted to higher temperatures, as a consequence of a positive $\kappa$ parameter. We define the following rescaling:

$$
\chi_{1}^{B^{(\text {toy })}}\left(T, \hat{\mu}_{B}\right)=\hat{\mu}_{B} \chi_{2}^{B}\left(T\left(1+\kappa \hat{\mu}_{B}^{2}\right), 0\right)
$$

The quality of the agreement between $\chi_{1}^{B^{\text {toy }}}$ and $\chi_{1}^{B}$ can be seen in the left panel of figure 2 . For the rescaling we used $\kappa=0.02$ as obtained for the $\mu_{S}=0$ setup in ref. [38]. We note that $\chi_{2}^{B}$ at finite chemical potential cannot be so simply approximated by shifting the $\mu_{B}=0$ result.

The relation (3.2) is just an approximation, but if it is takes as a definition of a toy model it gives access to the $\hat{\mu}_{B}$ dependence at any temperature if $\chi_{2}^{B}(T, 0)$ is known. The high $\hat{\mu}_{B}$-derivatives of $\chi_{1}^{B^{(\text {toy })}}$ lead to high $T$-derivatives of $\chi_{2}^{B}(T, 0)$, this is only possible if we have a smooth interpolation, preferably an analytical formula for $f(T)=\chi_{2}^{B}(T, 0)$. Being (3.2) inexact there is no point in tuning $f(T)$ too precisely to the lattice data, instead we choose a simple sigmoid fit function: $f(T)=A+B T+C \operatorname{atan}(D(T-E))$ that describes the gross features of the curve in the $[120: 300] \mathrm{MeV}$ temperature interval. The resulting $\chi_{2 n}^{B}(T, 0)$ functions we give later in figure 4 where we also compare this toy model to the lattice result.

Although the toy model only incorporates the feature of a smooth $\chi_{2}^{B}(T, 0)$ and the shifting of $T_{c}$ with the chemical potential it correctly reproduces the oscillatory pattern of the higher fluctuations.

At this point we use only the $\chi_{8}^{B} / \chi_{4}^{B}$ ratio to motivate a prior that we will introduce in the next section to stabilize the $\hat{\mu}_{B}$ fit. We know that in the Hadron Resonance Gas model this ratio is one. We must, however, expect also slightly higher values, that would 

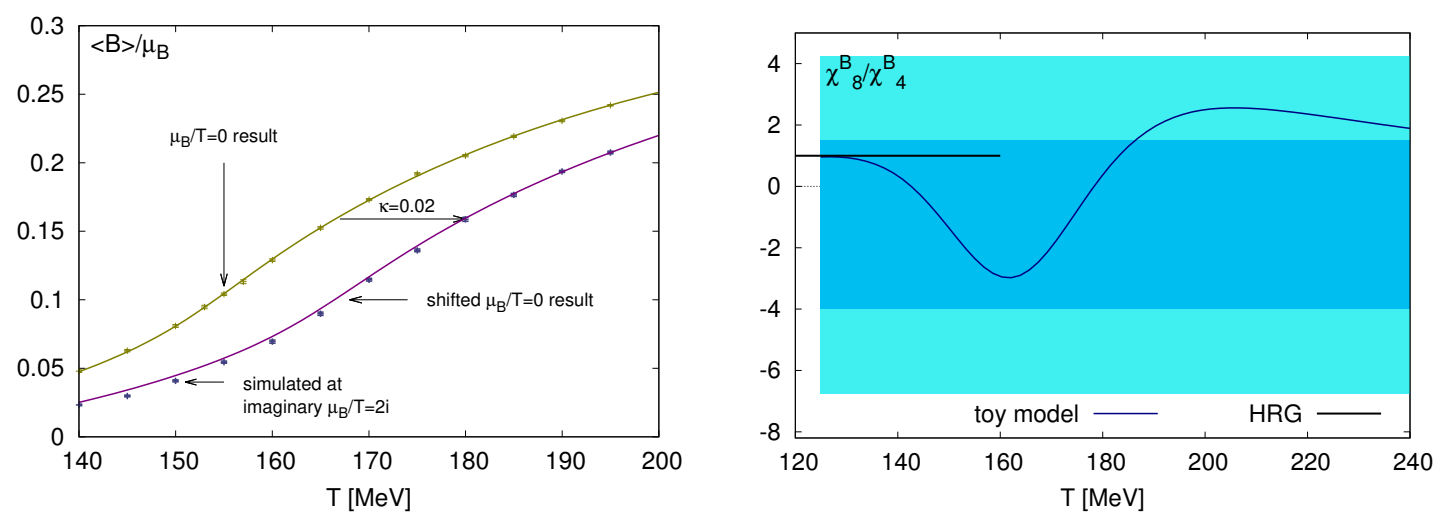

Figure 2. Toy model setup and its result for $\chi_{8}^{B} / \chi_{4}^{B}$. In the left panel we observe that the directly simulated $\chi_{1}^{B}\left(T, \hat{\mu}_{B}\right) / \mu_{B}$ at imaginary chemical potential can be approximately reproduced by rescaling the $\mu_{B}=0$ result $\chi_{2}^{B}(T, 0)$, (see eq. (3.2)). The right panel shows a specific ratio of high order fluctuations. The extraction of $\chi_{8}^{B}$ is stabilized by a prior bound to $\chi_{4}^{B}$. With dark and light bands we show the one and two $\sigma$ regions of the prior distribution. The Hadron Resonance Gas predicts 1 for this ratio, at high temperatures the Stefan-Boltzmann limit gives zero.

correspond to a signal from a nearby critical end point. The pattern of $\chi_{8}^{B} / \chi_{4}^{B}$ in the right panel of figure 2 is slightly asymmetric. In this paper we define a prior with

$$
\chi_{8}=\chi_{4}(-1.25+2.75 \xi)
$$

where $\xi$ is a stochastic variable with normal distribution.

The HRG prediction is $\chi_{2 n} / \chi_{4}=1$, which is within the prior range. At high temperatures higher order fluctuations quickly approach the Stefan-Boltzmann limit as it was seen in HTL perturbation theory [41, 42] as well as on lattice [28, 43]. The Stefan-Boltzmann limit is zero for $\chi_{2 n}^{B}$ if $n \geq 3$. There is no reason to expect our toy model to work at high temperatures, and indeed, the $\chi_{6}^{B}$ estimate in figure 2 converges to zero slower than the HTL prediction in ref. [42].

\subsection{Correlated fit with priors}

We start with the analysis for $\chi_{2}^{B}(T), \chi_{4}^{B}(T)$ and $\chi_{6}^{B}(T)$. Our goal is to calculate these quantities at zero chemical potential, using the imaginary chemical potential data up to $\chi_{4}^{B}\left(T, \hat{\mu}_{B}\right)$. In this work we extract these derivatives at a fixed temperature. Results for different temperatures are obtained completely independently, an interpolation in temperature is not necessary at any point. Thus, the error bars in our results plot will be independent. The errors between the quantities $\chi_{2}^{B}(T), \chi_{4}^{B}(T)$ and $\chi_{6}^{B}(T)$ will be highly correlated, though, since these are extracted through the same set of ensembles at the given temperature. This correlation will be taken into account when combined quantities are calculated, or when an extrapolation to real chemical potential is undertaken.

Thus we consider the ensembles at a fixed temperature $T$. For each value of imaginary $\mu_{B} \neq 0$ we determine $\chi_{1}^{B}, \chi_{2}^{B}, \chi_{3}^{B}$ and $\chi_{4}^{B}$ from simulation, while for $\mu_{B}=0$ only $\chi_{2}^{B}$ and $\chi_{4}^{B}$ can be used, since $\chi_{1}^{B}$ and $\chi_{3}^{B}$ are odd functions of $\mu_{B}$ and therefore equal to zero. 
We make the ansatz for the pressure:

$$
\chi_{0}^{B}\left(\hat{\mu}_{B}\right)=c_{0}+c_{2} \hat{\mu}_{B}^{2}+c_{4} \hat{\mu}_{B}^{4}+c_{6} \hat{\mu}_{B}^{6}+c_{8} \hat{\mu}_{B}^{8}+c_{10} \hat{\mu}_{B}^{10}
$$

where the Taylor expansion coefficients $c_{n}$ are related to the baryon number fluctuations $\chi_{n}^{B}$ by: $n ! c_{n}=\chi_{n}^{B}$. Our data do not allow for an independent determination of $c_{8}$ and $c_{10}$. Nevertheless, in order to have some control over these terms we make assumption on the higher order terms. In section 3.2 we have motivated and specified our choice for a prior distribution of eq. (3.3) for $\chi_{8}^{B}=8$ ! $c_{8}$. In the fit function we keep the terms up to $\chi_{10}^{B}=10 ! c_{10}$. Without this term the statistical errors on $\chi_{8}^{B}$ were clearly smaller, but the fit would be less controlled. As the highest order in the function, the resulting $\chi_{10}^{B}$ probably contains severe contamination from even higher order terms. For this reason, and since we fit $\chi_{10}^{B}$ with large statistical errors we do not give results on that quantity. For simplicity, we use the same prior distribution for $\chi_{10}^{B} / \chi_{4}^{B}$ as for $\chi_{8}^{B} / \chi_{4}^{B}$.

We can then rewrite our ansatz as

$$
\chi_{0}^{B}\left(\hat{\mu}_{B}\right)=c_{0}+c_{2} \hat{\mu}_{B}^{2}+c_{4} \hat{\mu}_{B}^{4}+c_{6} \hat{\mu}_{B}^{6}+\frac{4 !}{8 !} c_{4} \epsilon_{1} \hat{\mu}_{B}^{8}+\frac{4 !}{10 !} c_{4} \epsilon_{2} \hat{\mu}_{B}^{10},
$$

where $\epsilon_{1}$ and $\epsilon_{2}$ are drawn randomly from a normal distribution with mean -1.25 and variance 2.75 . We use the same distribution for all temperatures. In effect, our $c_{8}$ and $c_{10}$ coefficients are stochastic variables. The used distribution for $\epsilon_{1,2}$ actually implements a prior for $\chi_{8}^{B}$ and $\chi_{10}^{B}$.

For this ansatz we calculate the following derivatives, which are the actually simulated lattice observables:

$$
\begin{aligned}
& \chi_{1}^{B}\left(\hat{\mu}_{B}\right)=2 c_{2} \hat{\mu}_{B}+4 c_{4} \hat{\mu}_{B}^{3}+6 c_{6} \hat{\mu}_{B}^{5}+\frac{4 !}{7 !} c_{4} \epsilon_{1} \hat{\mu}_{B}^{7}+\frac{4 !}{9 !} c_{4} \epsilon_{2} \hat{\mu}_{B}^{9} \\
& \chi_{2}^{B}\left(\hat{\mu}_{B}\right)=2 c_{2}+12 c_{4} \hat{\mu}_{B}^{2}+30 c_{6} \hat{\mu}_{B}^{4}+\frac{4 !}{6 !} c_{4} \epsilon_{1} \hat{\mu}_{B}^{6}+\frac{4 !}{8 !} c_{4} \epsilon_{2} \hat{\mu}_{B}^{8} \\
& \chi_{3}^{B}\left(\hat{\mu}_{B}\right)=24 c_{4} \hat{\mu}_{B}+120 c_{6} \hat{\mu}_{B}^{3}+\frac{4 !}{5 !} c_{4} \epsilon_{1} \hat{\mu}_{B}^{5}+\frac{4 !}{7 !} c_{4} \epsilon_{2} \hat{\mu}_{B}^{7} \\
& \chi_{4}^{B}\left(\hat{\mu}_{B}\right)=24 c_{4}+360 c_{6} \hat{\mu}_{B}^{2}+c_{4} \epsilon_{1} \hat{\mu}_{B}^{4}+\frac{4 !}{6 !} c_{4} \epsilon_{2} \hat{\mu}_{B}^{6} .
\end{aligned}
$$

We perform a correlated fit for the four measured observables, thus obtaining the values of $c_{2}, c_{4}$ and $c_{6}$ for each temperature, and the corresponding $\chi_{2}^{B}, \chi_{4}^{B}$ and $\chi_{6}^{B}$. We repeat the fit for 1000 random draws for $\epsilon_{1}$ and $\epsilon_{2}$. The result is weighted using the Akaike Information Criterion [44]. Through these weights we get a posterior distribution from the prior distribution. Our final estimate for $\chi_{8}^{B}$ represents this posterior distribution. These results are shown in figure 3 , together with an estimate of $\chi_{8}^{B}$, related to $\chi_{4}^{B}$ by eq. (3.5).

Finally we show a comparison of these fit results to the toy model that we introduced in section 3.2. We find a reasonable (though not complete) agreement with data. The toy model correctly finds the zero crossings and acceptably approximates heights of the peaks in the temperature dependence. (Note, that varying the interpolator in the toy model will alter the peak heights of the resulting $\chi_{2 n}^{B}(T)$ functions.) We emphasize that the toy model assumed only the shift of the transition temperature with the chemical potential 

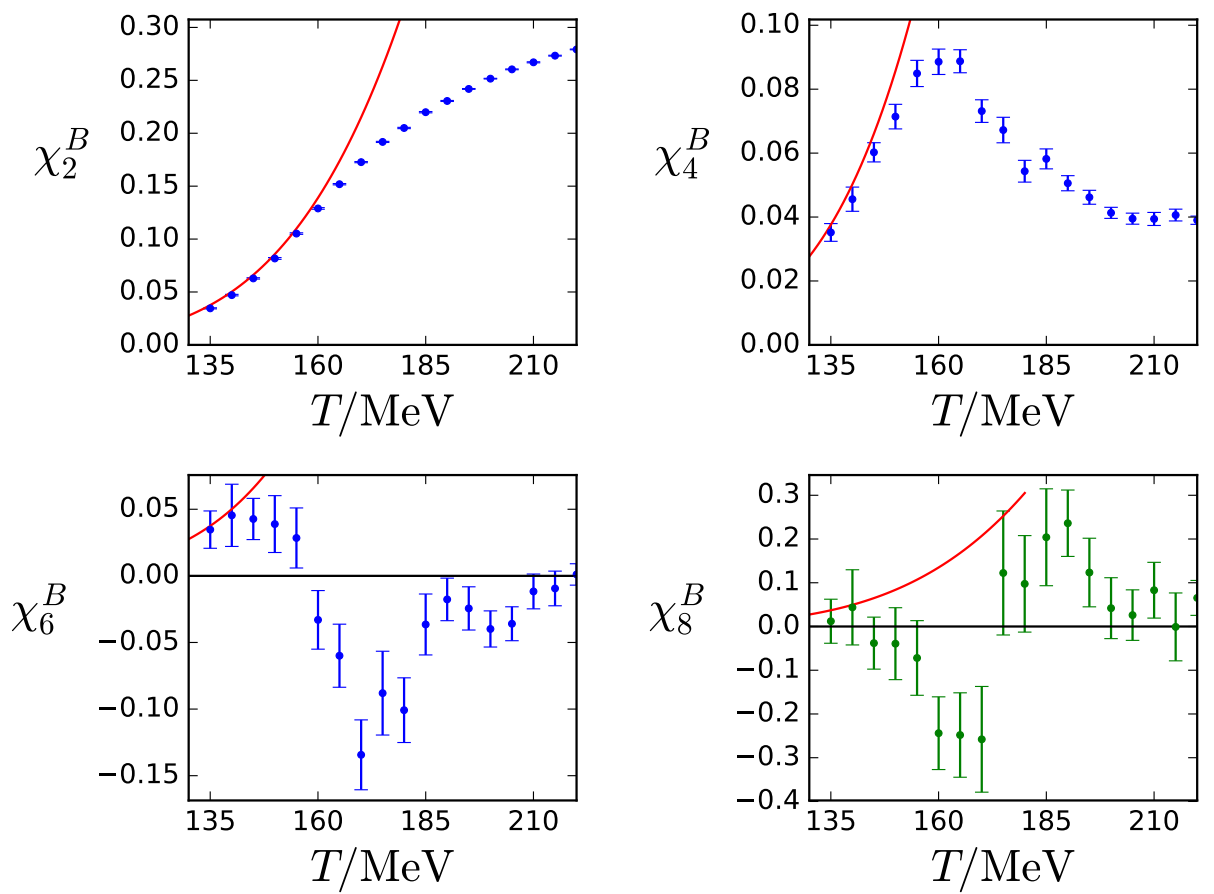

Figure 3. Results for $\chi_{2}^{B}, \chi_{4}^{B}, \chi_{6}^{B}$ and an estimate for $\chi_{8}^{B}$ as functions of the temperature, obtained from the single-temperature analysis. We plot $\chi_{8}^{B}$ in green to point out that its determination is guided by a prior, which is linked to the $\chi_{4}^{B}$ observable by eq. (3.5). The red curve in each panel corresponds to the Hadron Resonance Gas (HRG) model result.

while keeping the strength of the transition constant. If this toy model is in agreement with the lattice data, then the lattice data are also compatible with a scenario without a critical end point.

\subsection{Cross-correlators}

So far we only considered derivatives with respect to the baryonic chemical potential. In our previous, direct analysis in ref. [28], the $\mu_{B}$-derivatives had larger errors than $\mu_{Q}-$ or $\mu_{S}$-derivatives. For $\mu_{Q}$, the most noisy disconnected contributions come with smaller prefactors, while for $\mu_{S}$ the disconnected contributions are small due to the heavier strange mass. Our approach was designed to improve the $\mu_{B}$-derivatives only. Therefore, the $\mu_{S}$ and $\mu_{Q}$ derivatives have to be simulated directly and without the support from the fit that we used in the $\mu_{B}$ direction. Our result on $\chi_{j k}^{Q S}$ improved only due to the increase in the statistics since [28].

On the other hand, baryon-strange and baryon-charge mixed derivatives do benefit from the imaginary $\mu_{B}$ data. We simulate various $\chi_{i, j, k}^{B, Q, S}$ with the appropriate values of $j$ and $k$ and all possible values of $i$ so that $i+j+k \leq 4$. For each group of fluctuations with the same $j$ and $k$ we perform a fit analogous to the procedure described in section 3.3.

Let's take the example of $j=1, k=0$. Our ansatz for cross-correlators is analogous to eqs. (3.6)-(3.9):

$$
\chi_{01}^{B S}\left(\hat{\mu}_{B}\right)=\chi_{11}^{B S} \hat{\mu}_{B}+\frac{1}{3 !} \chi_{31}^{B S} \hat{\mu}_{B}^{3}+\frac{1}{5 !} \chi_{51}^{B S} \hat{\mu}_{B}^{5}+\frac{1}{7 !} \chi_{71}^{B S} \hat{\mu}_{B}^{7}+\frac{1}{9 !} \chi_{91}^{B S} \hat{\mu}_{B}^{9}
$$



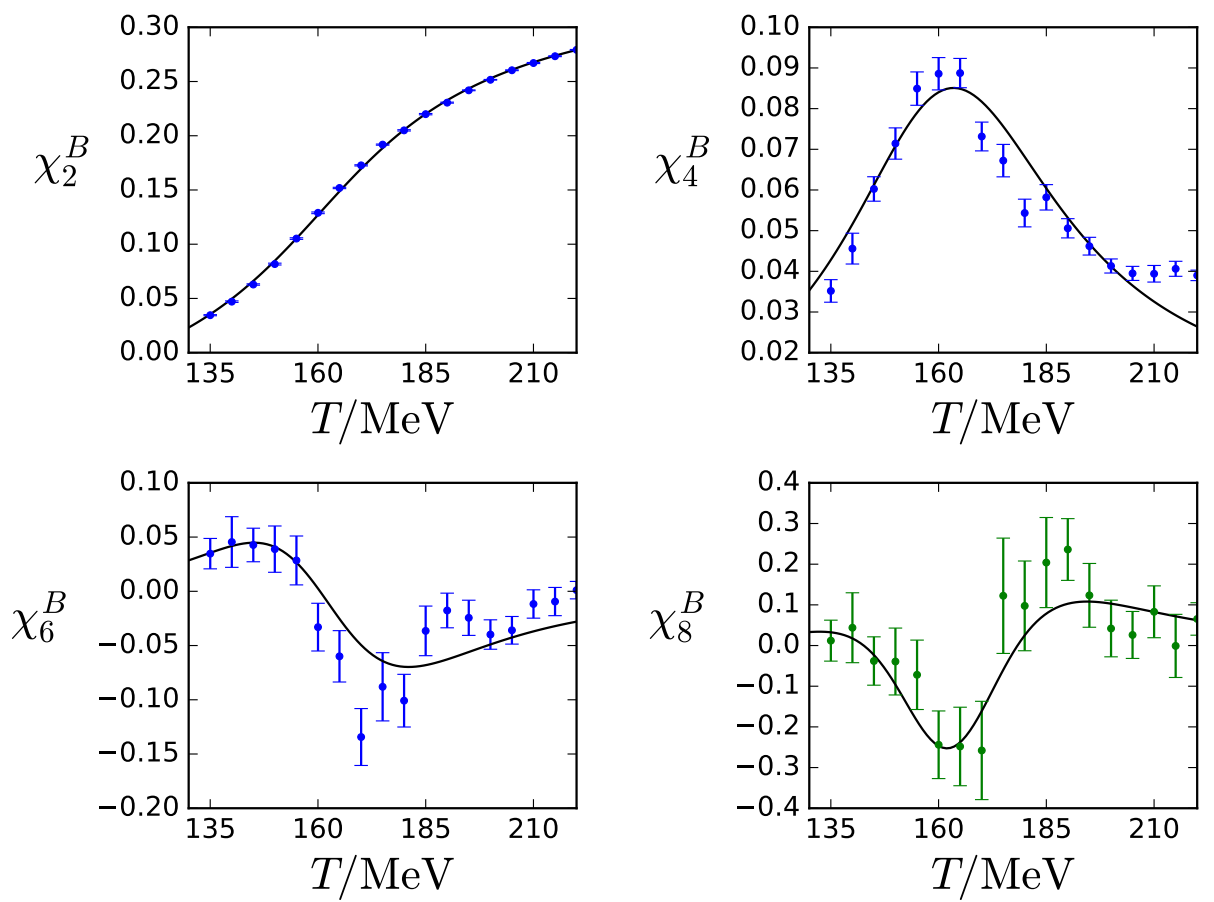

Figure 4. Results for $\chi_{2}^{B}, \chi_{4}^{B}, \chi_{6}^{B}$ and an estimate for $\chi_{8}^{B}$ as functions of the temperature shown together with the result of the toy model of section 3.2. The toy model reproduces much of the features of the lattice result.

We truncated the expression at tenth order. The priors assume $\left|\chi_{71}^{B S}\right| \lesssim\left|\chi_{31}^{B S}\right|$ and $\left|\chi_{91}^{B S}\right| \lesssim\left|\chi_{31}^{B S}\right|$, as it is certainly true at high temperature and within the HRG model. The prior distribution is wider than 1 , we used the same mean and variance as in the channel with no $\mu_{S}$ derivative.

When we use eq. (3.10) we take $\chi_{1}^{S}, \chi_{11}^{B S}, \chi_{21}^{B S}$ and $\chi_{31}^{B S}$ as correlated quartets for each imaginary chemical potential and determine the three free coefficients of eq. (3.10). This fitting procedure is repeated 1000 times with random $\chi_{71}^{B S} / \chi_{31}^{B S}$ and $\chi_{91}^{B S} / \chi_{31}^{B S}$ coefficients. Again, using the Akaike weights we constrain the prior distribution. The resulting estimate for $\chi_{71}^{B S}$ along with the fit coefficients are shown in figure 5. The posterior for $\chi_{91}^{B S}$ is not only noisy, but it is probably heavily contaminated by the higher orders that we did not account for.

The other channels with higher $\mu_{S}$ or $\mu_{Q}$ derivatives are obtained analogously. These are plotted in appendix A.

\subsection{Error analysis}

For a reliable comparison between experimental measurements and theoretical calculations, the error estimate is an important ingredient. Our statistical error is estimated through the jackknife method. For our systematic error there are several sources. We determine our systematic error by the histogram method described in [45], where each analysis is weighted with the Akaike information criteria. We include the influence of the number of points in the $\mu_{B}$ direction, by either including or ignoring the data from our highest 

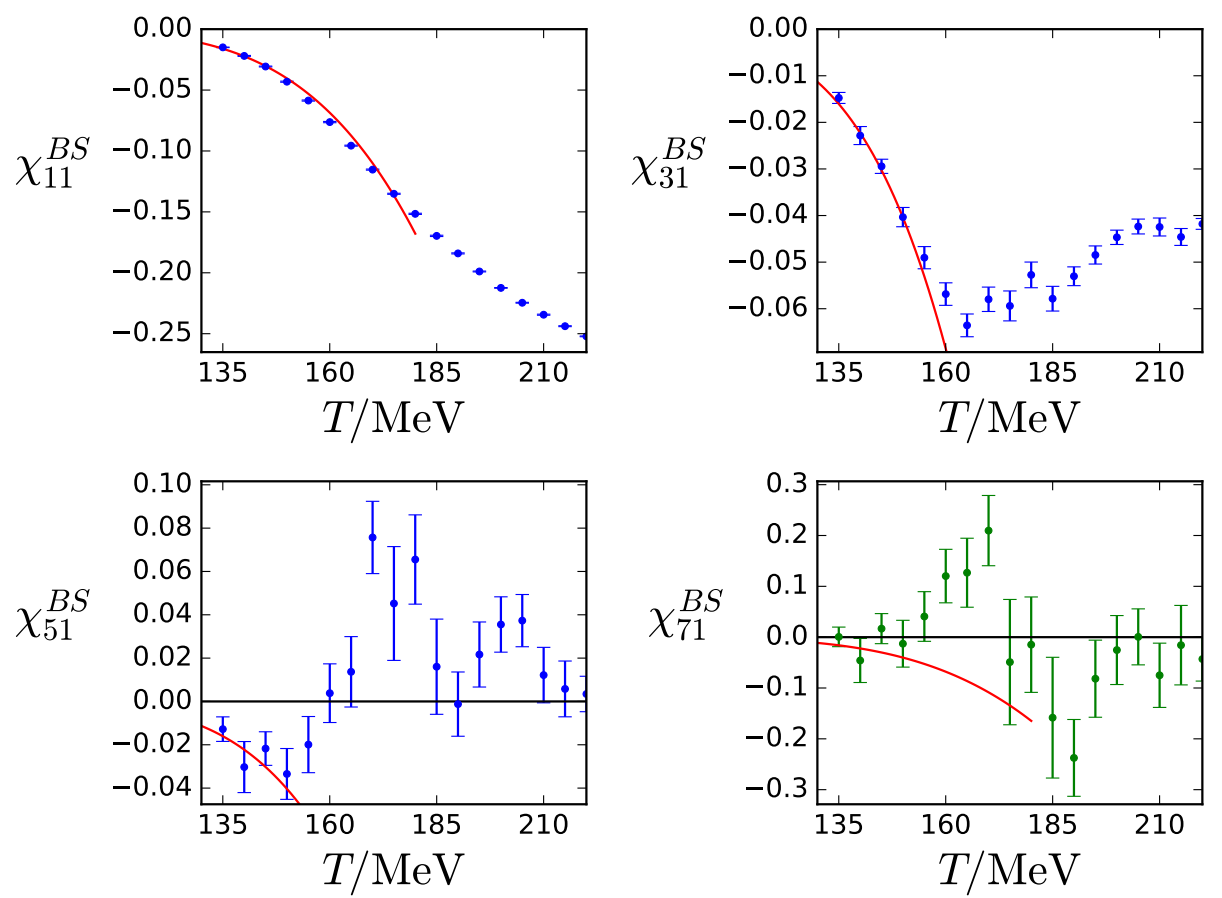

Figure 5. $\chi_{11}^{B S}, \chi_{31}^{B S}, \chi_{51}^{B S}$ and an estimate for $\chi_{71}^{B S}$ as functions of the temperature. The red curves are the HRG model results.

value of $\mu_{B}$. A very important source for our systematic error is the influence of the higher order contributions in $\mu_{B}$. This effect was estimated by adding the higher order terms with pre-factors $\epsilon_{1}$ and $\epsilon_{2}$ as described in section 3.3. We consider 1000 different $\epsilon$ pairs and add the different analyses to our histogram. The width of the histogram using Akaike weights corresponding to the fit quality gives the systematic errors for the fit coefficients, and from the same histogram we obtain the posterior distributions for $\epsilon_{1}$. The physical quantities that are constrained only by the posterior distribution are plotted with green symbols.

These histograms are built independently for each number $\left(j\right.$ and $k$ ) of $\mu_{S}$ and $\mu_{Q}$ derivatives. When calculating the systematics for the cumulant ratios (section 4) we need to calculate different combinations of diagonal and non-diagonal fluctuations from the available analyses. Though these fits (corresponding to the same temperature) are carried out separately we keep track of the statistical correlation, by maintaining the jackknife ensembles throughout the analysis. The correct propagation of systematic errors is a more elaborate procedure. When $\chi_{i j k}^{B S Q}$ coefficients are combined with different $j, k$ pairs, different histograms have to be combined. If we had only two variables to combine, each of the 2000 first fit variants should be combined with each of the 2000 second fit variants and use the product of the respective probability weights. Instead, we combine the fit results by drawing 'good' fits by importance sampling from each histogram independently. In this way, $\mathcal{O}(100)$ random combinations of $\chi_{i j k}^{B S Q}$ results already give convergence for each discussed quantity and its error bar. For the results in this paper we used 1000 such random combinations. This procedure assumes that between different $j, k$ pairs the prior distribution is uncorrelated. 


\section{Phenomenology at finite chemical potential}

For a comparison with heavy ion collision experiments, the cumulants of the net-baryon distribution are very useful observables. The first four cumulants are the mean $M_{B}$, the variance $\sigma_{B}^{2}$, the skewness $S_{B}$ and the kurtosis $\kappa_{B}$. By forming appropriate ratios, we can cancel out explicit volume factors. However, the measured distributions themselves may still depend on the volume, which one should take into account when comparing to experiments.

Heavy ion collisions involving lead or gold atoms at $\mu_{B}>0$ correspond to the following situation

$$
\left\langle n_{S}\right\rangle=0 \quad\left\langle n_{Q}\right\rangle=0.4\left\langle n_{B}\right\rangle .
$$

For each $T$ and $\mu_{B}$ pair, we have to first calculate $\mu_{Q}$ and $\mu_{S}$ that satisfy this condition. The resulting $\mu_{Q}\left(\mu_{B}\right)$ and $\mu_{S}\left(\mu_{B}\right)$ functions, too, can be Taylor expandend [19, 20], introducing

$$
\begin{aligned}
q_{j} & =\left.\frac{1}{j !} \frac{d^{j} \hat{\mu}_{Q}}{\left(d \hat{\mu}_{B}\right)^{j}}\right|_{\mu_{B}=0} \\
s_{j} & =\left.\frac{1}{j !} \frac{d^{j} \hat{\mu}_{S}}{\left(d \hat{\mu}_{B}\right)^{j}}\right|_{\mu_{B}=0} .
\end{aligned}
$$

We investigate three different ratios of cumulants:

$$
\begin{aligned}
\frac{M_{B}}{\sigma_{B}^{2}} & =\frac{\chi_{1}^{B}\left(T, \hat{\mu}_{B}\right)}{\chi_{2}^{B}\left(T, \hat{\mu}_{B}\right)}=\hat{\mu}_{B} r_{12}^{B, 1}+\hat{\mu}_{B}^{3} r_{12}^{B, 3}+\ldots \\
\frac{S_{B} \sigma_{B}^{3}}{M_{B}} & =\frac{\chi_{3}^{B}\left(T, \hat{\mu}_{B}\right)}{\chi_{1}^{B}\left(T, \hat{\mu}_{B}\right)}=r_{31}^{B, 0}+\hat{\mu}_{B}^{2} r_{31}^{B, 2}+\ldots \\
\kappa_{B} \sigma_{B}^{2} & =\frac{\chi_{4}^{B}\left(T, \hat{\mu}_{B}\right)}{\chi_{2}^{B}\left(T, \hat{\mu}_{B}\right)}=r_{42}^{B, 0}+\hat{\mu}_{B}^{2} r_{42}^{B, 2}+\hat{\mu}_{B}^{4} r_{42}^{B, 4}+\ldots
\end{aligned}
$$

The $\mu_{B}$-dependence of the $\chi_{i}^{B}\left(T, \hat{\mu}_{B}\right)$ can again be written as a Taylor series:

$$
\begin{aligned}
\chi_{i, j, k}^{B Q S}\left(\hat{\mu}_{B}\right)= & \chi_{i, j, k}^{B Q S}(0)+\hat{\mu}_{B}\left[\chi_{i+1, j, k}^{B Q S}(0)+q_{1} \chi_{i, j+1, k}^{B Q S}(0)+s_{1} \chi_{i, j, k+1}^{B Q S}(0)\right] \\
& +\frac{1}{2} \hat{\mu}_{B}^{2}\left[\chi_{i+2, j, k}^{B Q S}(0)+q_{1}^{2} \chi_{i, j+2, k}^{B Q S}(0)+s_{1}^{2} \chi_{i, j, k+2}^{B Q S}(0)\right. \\
& \left.+2 q_{1} s_{1} \chi_{i, j+1, k+1}^{B Q S}(0)+2 q_{1} \chi_{i+1, j+1, k}^{B Q S}(0)+2 s_{1} \chi_{i+1, j, k+1}^{B Q S}(0)\right]+\ldots
\end{aligned}
$$

The $\chi$ coefficients that we determined in section 3 include derivatives up to sixth order, and we have estimates for the eighth order, too. The fit coefficients corresponding to the tenth order are likely to be contaminated by higher orders, that we did not include into the ansatz. These $\chi_{i j k}^{B Q S}$ coefficients, however, are given for $j+k \leq 4$, which is the highest order that we used in $\mu_{Q}$ and $\mu_{S}$. 

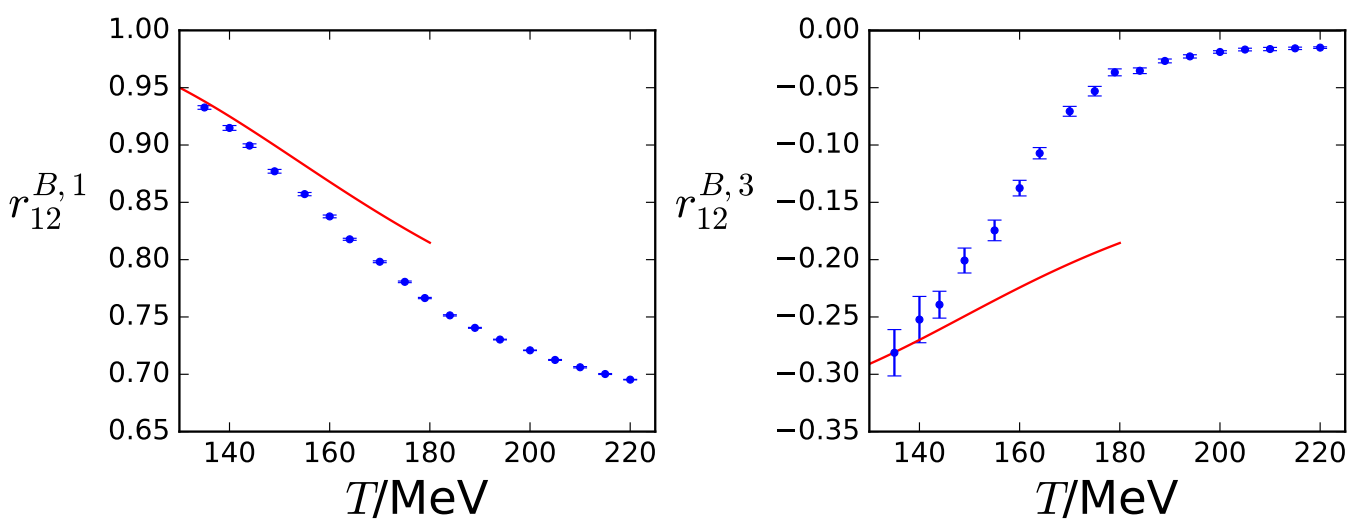

Figure 6. Taylor expansion coefficients for $\frac{M_{B}}{\sigma_{B}^{2}}=\frac{\chi_{1}^{B}\left(T, \hat{\mu}_{B}\right)}{\chi_{2}^{B}\left(T, \hat{\mu}_{B}\right)}$ as functions of the temperature: $r_{12}^{B, 1}$ (left panel) and $r_{12}^{B, 3}$ (right panel).
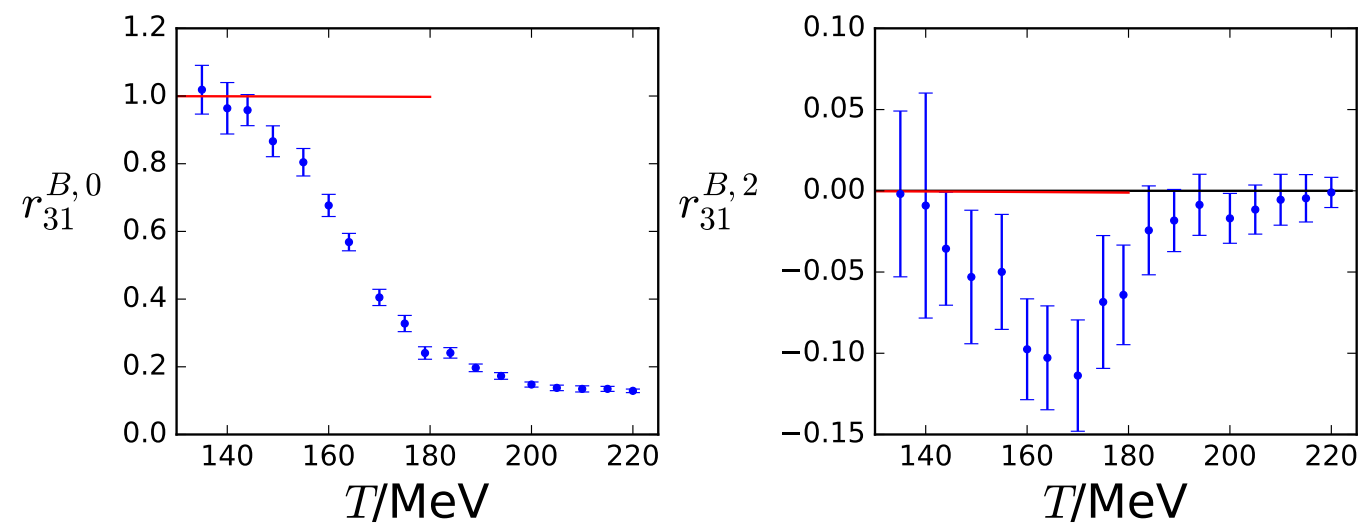

Figure 7. Taylor expansion coefficients for $\frac{S_{B} \sigma_{B}^{3}}{M_{B}}=\frac{\chi_{3}^{B}\left(T, \hat{\mu}_{B}\right)}{\chi_{1}^{B}\left(T, \hat{\mu}_{B}\right)}$ as functions of the temperature: $r_{31}^{B, 0}$ (left panel) and $r_{31}^{B, 2}$ (right panel).

This list of coefficients allows us to calculate the $r_{i j}^{B, k}$ coefficients from equations (4.4), (4.5) and (4.6). The results for the $r_{i j}^{B, k}$ coefficients are shown in figures 6,7 and 8 . We confirm the observation from ref. [23] that the coefficient $r_{42}^{B, 2}$ has a similar temperature dependence as $r_{31}^{B, 2}$ but it is $\sim 3$ times larger in magnitude.

For higher order coefficients, higher order derivatives in $\mu_{S}$ and $\mu_{Q}$ are needed. The direct simulations have a rapidly increasing error with the order of the derivative, and very large statistics would be needed to improve our calculations at this point. Another possibility would be to simulate new ensembles with finite $\mu_{S}$ and $\mu_{Q}$ and do a similar fit as for the $\mu_{B}$ direction. This approach has been used in [27].

After calculating the Taylor coefficients for $S_{B} \sigma_{B}^{3} / M_{B}$ and $\kappa_{B} \sigma_{B}^{2}$, we use these results to extrapolate these quantities to finite chemical potential. They are shown in figure 9 . In the left panel, $S_{B} \sigma_{B}^{3} / M_{B}$ is shown as a function of the chemical potential for different temperatures. The Taylor expansion for this quantity is truncated at $\mathcal{O}\left(\hat{\mu}_{B}^{2}\right)$. The black points in the figure are the experimental results from the STAR collaboration from an analysis of cumulant ratios measured at mid-rapidity, $|y| \leq 0.5$, including protons and anti-protons 

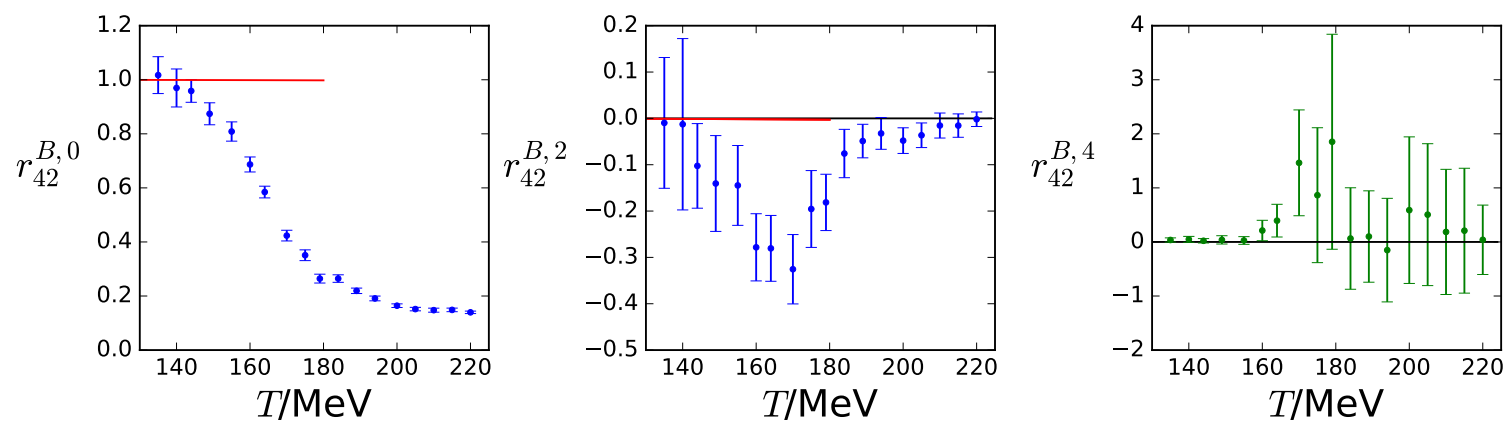

Figure 8. Taylor expansion coefficients for $\kappa_{B} \sigma_{B}^{2}=\frac{\chi_{4}^{B}\left(T, \hat{\mu}_{B}\right)}{\chi_{2}^{B}\left(T, \hat{\mu}_{B}\right)}$ as functions of the temperature: $r_{42}^{B, 0}$ (left panel) $r_{42}^{B, 2}$ (middle panel), $r_{42}^{B, 4}$ (right panel). The latter is not obtained independently, but by means of the prior ansatz (see text): for this reason, we plot it in green.
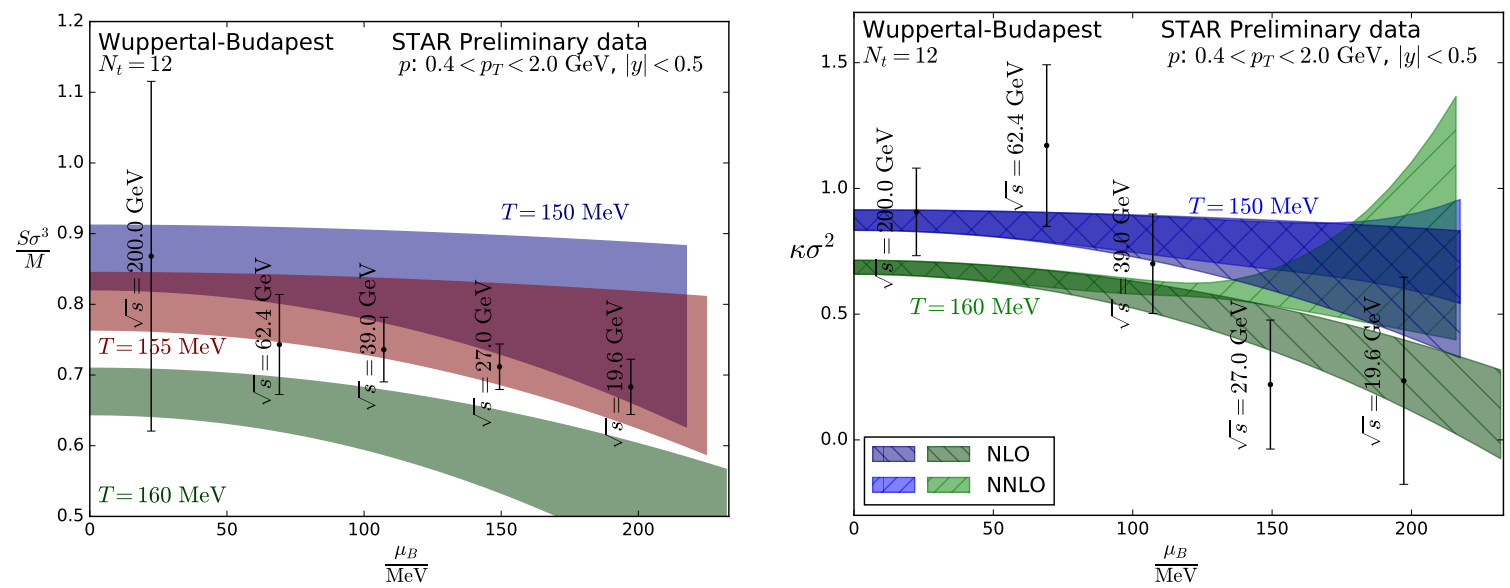

Figure 9. $S_{B} \sigma_{B}^{3} / M_{B}$ (left panel) and $\kappa_{B} \sigma_{B}^{2}$ (right panel) extrapolated to finite chemical potential. The left panel is extrapolated up to $\mathcal{O}\left(\hat{\mu}_{B}^{2}\right)$. In the right panel, the darker bands correspond to the extrapolation up to $\mathcal{O}\left(\hat{\mu}_{B}^{2}\right)$, whereas the lighter bands also include the $\mathcal{O}\left(\hat{\mu}_{B}^{4}\right)$ term.

with transverse momenta $0.4 \mathrm{GeV} \leq p_{t} \leq 2.0 \mathrm{GeV}[46,47]$. The beam energies were translated to chemical potentials using the fitted formula of ref. [48]. Even if we do not quantitatively compare the lattice bands to the measurements to extract the freeze-out parameters, as experimental higher order fluctuations might be affected by several effects of non-thermal origin and our lattice results are not continuum extrapolated, we notice that the trend of the data with increasing $\mu_{B}$ can be understood in terms of our Taylor expansion.

In the right panel, we show $\kappa_{B} \sigma_{B}^{2}$ as a function of $\mu_{B} / T$ for different temperatures. The darker bands correspond to the extrapolation up to $\mathcal{O}\left(\hat{\mu}_{B}^{2}\right)$, whereas the lighter bands also include the $\mathcal{O}\left(\hat{\mu}_{B}^{4}\right)$ term. Also in this case, the black points are the experimental results from the STAR collaboration with transverse momentum cut $0.4 \mathrm{GeV} \leq p_{t} \leq 2.0 \mathrm{GeV}[46,47]$. By comparing the two different truncations of the Taylor series we can conclude that, as we increase the temperature, the range of applicability of our Taylor series decreases: while at $T=150 \mathrm{MeV}$ the two orders agree in the whole $\mu_{B} / T$ range shown in the figure, at $T=160 \mathrm{MeV}$ the central line of the next-to-next-to-leading order (NNLO) bends upwards 
and is not contained in the next-to-leading order (NLO) band. This behaviour reflects the pattern in the Taylor coefficients of figure 8. Notice that up to $T=155 \mathrm{MeV} r_{42}^{B, 4}$ is consistent to zero, and already positive at $160 \mathrm{MeV}$ or somewhat higher temperatures. For temperatures where we have a small $r_{42}^{B, 4}$ coefficient the NLO result is satisfactory. To make the NNLO prediction precise substantially more computer time would be needed.

\section{Conclusions and outlook}

In this manuscript, we have calculated several diagonal and non-diagonal fluctuations of electric charge, baryon number and strangeness up to sixth-order, in a system of $2+1+1$ quark flavors with physical quark masses, on a lattice with size $48^{3} \times 12$. The analysis has been performed simulating the lower order fluctuations at zero and imaginary chemical potential $\mu_{B}$, and extracting the higher order fluctuations as derivatives of the lower order ones at $\mu_{B}=0$. The chemical potentials for electric charge and strangeness have both been set to zero in the simulations. From these fluctuations, we have constructed ratios of baryon number cumulants as functions of $T$ and $\mu_{B}$, by means of a Taylor series which takes into account the experimental constraints $\left\langle n_{S}\right\rangle=0$ and $\left\langle n_{Q}\right\rangle=0.4\left\langle n_{B}\right\rangle$. These ratios qualitatively explain the behavior observed in the experimental measurements by the STAR collaboration as functions of the collision energy.

We focused on observables (baryon distribution, ratios of cumulants) that are less sensitive to lattice artefacts. An obvious extension of our work will be the use of finer lattices and a continuum extrapolation. The other extension is to use a two- or even three-dimensional mapping of the space of the imaginary chemical potentials using nonvanishing $\mu_{S}$ and $\mu_{Q}$. That would not only improve the $\mu_{S}-$ and $\mu_{Q}$-derivatives, but would allow us to study the melting of states with various strangeness and electric charge quantum numbers. Our first study in this direction using strangeness chemical potentials was published in ref. [49].

\section{Acknowledgments}

This project was funded by the DFG grant SFB/TR55. This work was supported by the Hungarian National Research, Development and Innovation Office, NKFIH grants KKP126769 and K113034. An award of computer time was provided by the INCITE program. This research used resources of the Argonne Leadership Computing Facility, which is a DOE Office of Science User Facility supported under Contract DE-AC0206CH11357. The authors gratefully acknowledge the Gauss Centre for Supercomputing e.V. (www.gauss-centre.eu) for funding this project by providing computing time on the GCS Supercomputer JUQUEEN [50] at Jülich Supercomputing Centre (JSC) as well as on HAZELHEN at HLRS Stuttgart, Germany. This material is based upon work supported by the National Science Foundation under grants no. PHY-1654219 and OAC-1531814 and by the U.S. Department of Energy, Office of Science, Office of Nuclear Physics, within the framework of the Beam Energy Scan Theory (BEST) Topical Collaboration. C.R. also acknowledges the support from the Center of Advanced Computing and Data Systems at the University of Houston. 

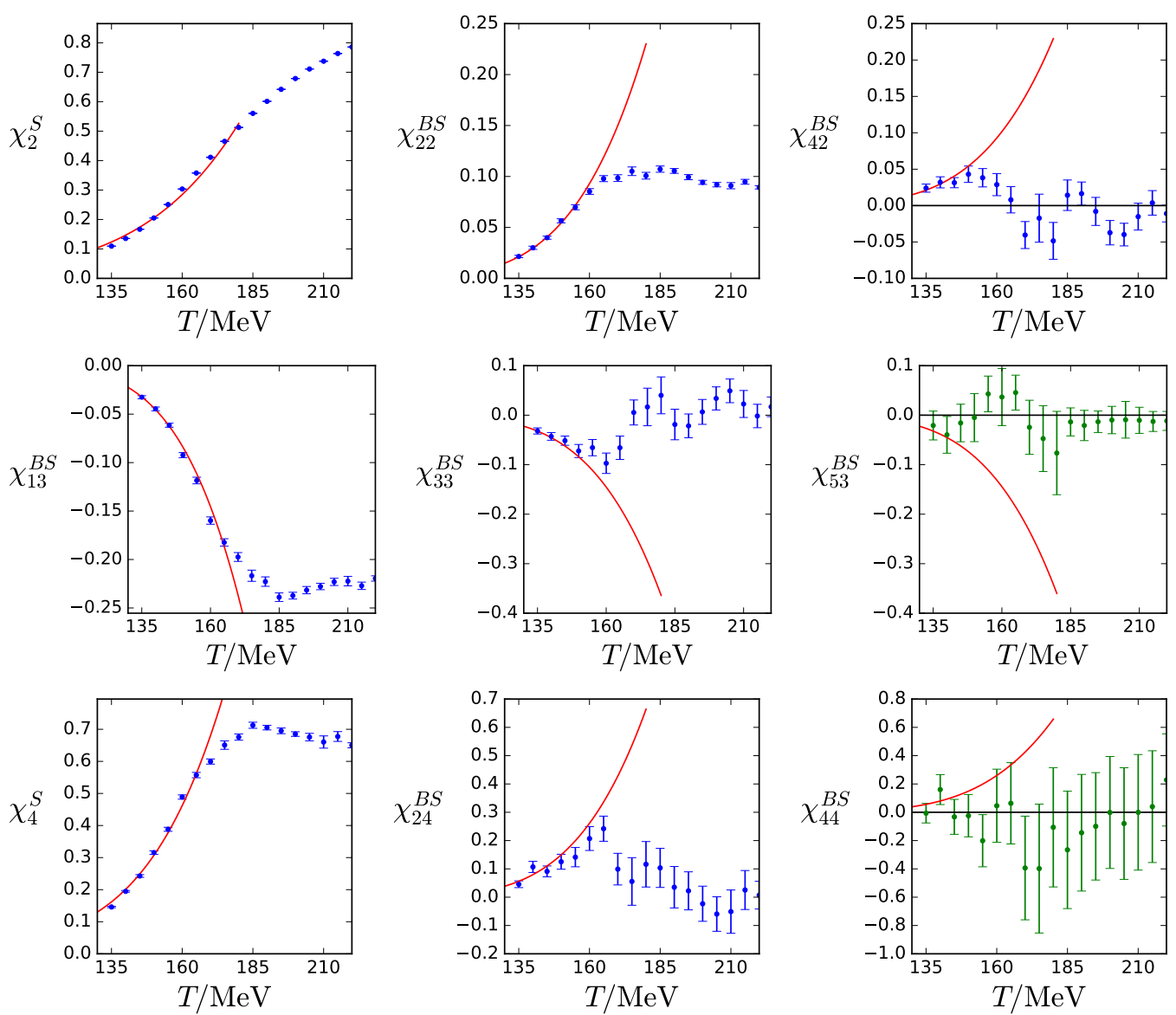

Figure 10. Results containing no electric charge derivative on the various correlators on our $48^{3} \times 12$ lattice as functions of the temperature. Green data points denote our estimates for the high orders, these were fitted using a prior distribution. The red curves are the HRG model results.

\section{A Results for the correlators}

In this appendix we present the non-diagonal fluctuations of conserved charges needed to construct the cumulant ratios at finite chemical potential $\mu_{B}$, satisfying the constraints $\left\langle n_{S}\right\rangle=0$ and $\left\langle n_{Q}\right\rangle=0.4\left\langle n_{B}\right\rangle$.

Like we did for the diagonal $\chi_{i}^{B}$, we simulate lower order fluctuations at finite imaginary chemical potential and extract the higher order fluctuations as derivatives of the lower order ones at $\mu_{B}=0$ : in particular, we simulate various $\chi_{i, j, k}^{B, Q, S}$ with the appropriate values of $j$ and $k$ and all possible values for $i$ so that

$$
i+j+k \leq 4,
$$

and extract the corresponding $\chi_{i, j, k}^{B, Q, S}$ with $i+j+k \leq 6$ and an estimate for $i+j+k=8$ and sometimes even $i+j+k=10$. By estimate (shown in green) we mean the posterior distribution that we get for the two highest orders when using priors, as discussed in the main text. In total we need 15 channels to obtain all the necessary terms.

In the following plots we show these results organized by the number of charge derivatives $(j)$ in figures 10-14. It is notoriously difficult to calculate charge correlators using 

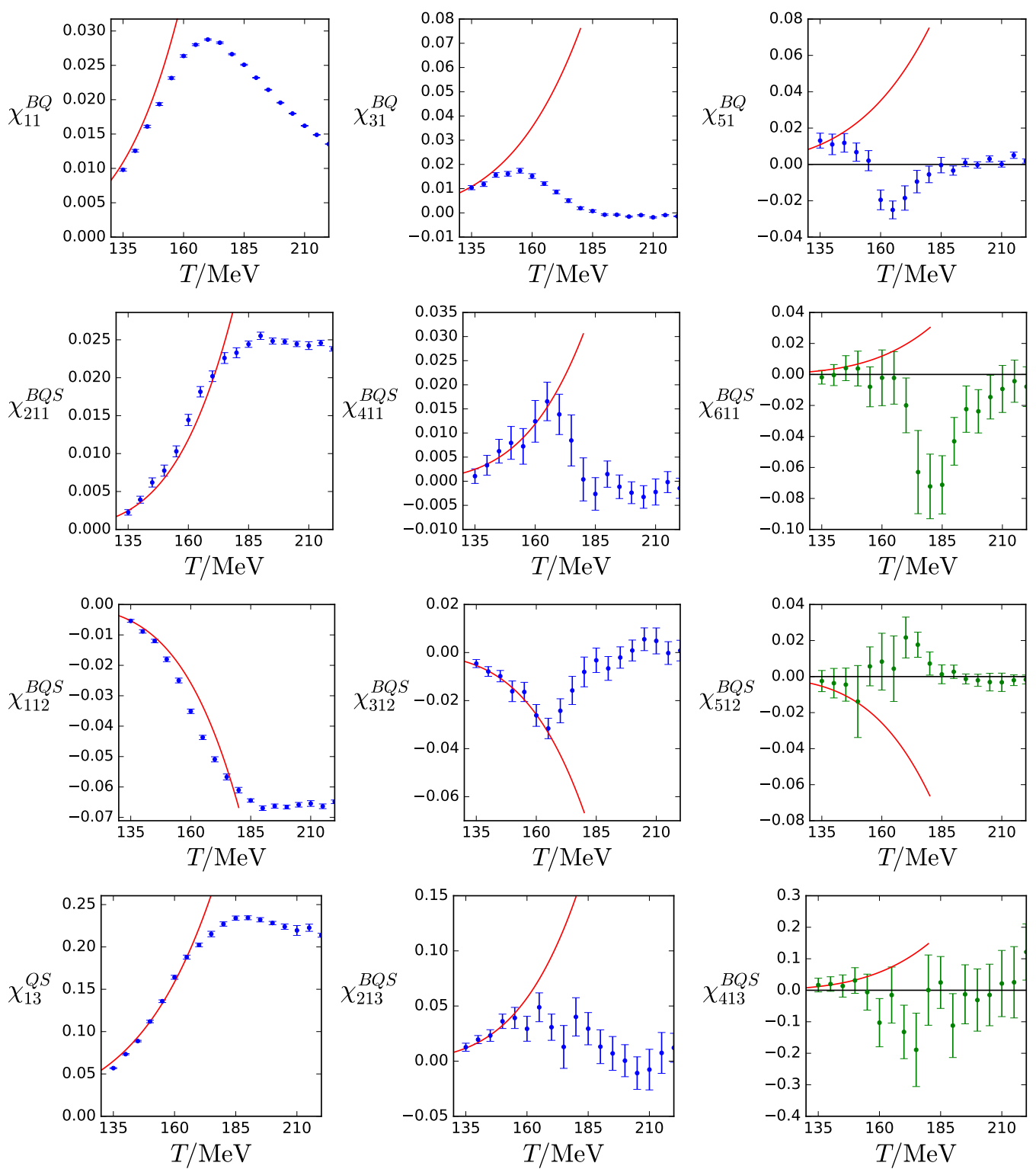

Figure 11. Results containing one electric charge derivative on the various correlators on our $48^{3} \times 12$ lattice as functions of the temperature. Green data points denote our estimates for the high orders, these were fitted using a prior distribution. The red curves are the HRG model results.

staggered fermions [28]. Correlators that are not protected by a baryon derivative are affected by significant discretization errors. It is understood in the HRG model context that discretization errors mostly affect the contributions from pions and kaons. Staggered lattice effects introduce the highest relative errors for the lightest mesons. Luckily, however, quantities with such discretization effects come with a small pre-factor into the final formulas of eqs. (4.4)-(4.6). If we had a complete isospin symmetry (factor 0.5 between $\left\langle n_{Q}\right\rangle$ and $\left\langle n_{B}\right\rangle$ in eq. (4.1)) then electric charge correlators would play no role at all in the extrapolation of baryon fluctuations. 

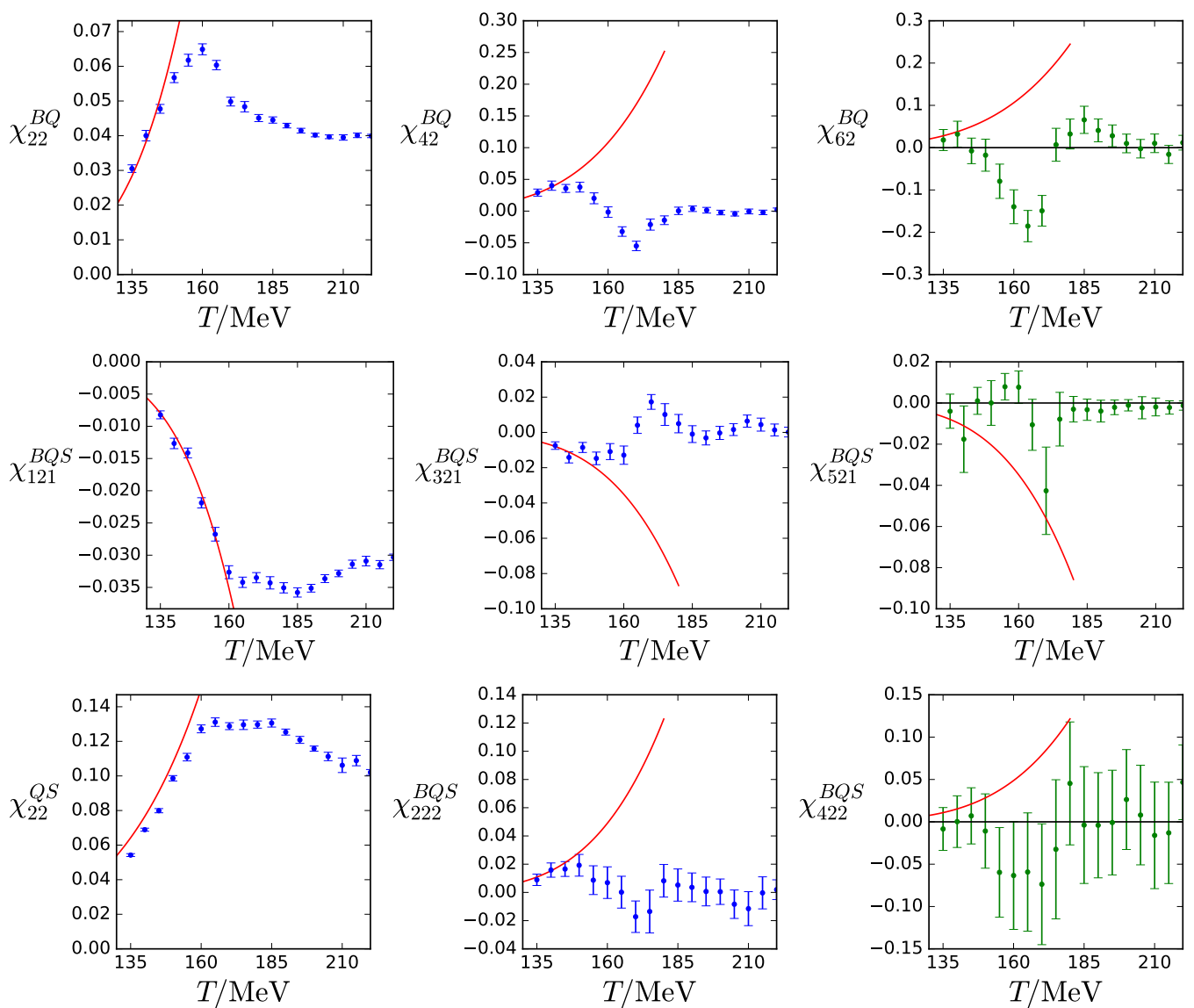

Figure 12. Results containing two electric charge derivatives on the various correlators on our $48^{3} \times 12$ lattice as functions of the temperature. Green data points denote our estimates for the high orders, these were fitted using a prior distribution. The red curves are the HRG model results. Charge correlators without baryon derivative (here $\chi_{22}^{Q S}$ ) are expected to have significant discretization errors. 

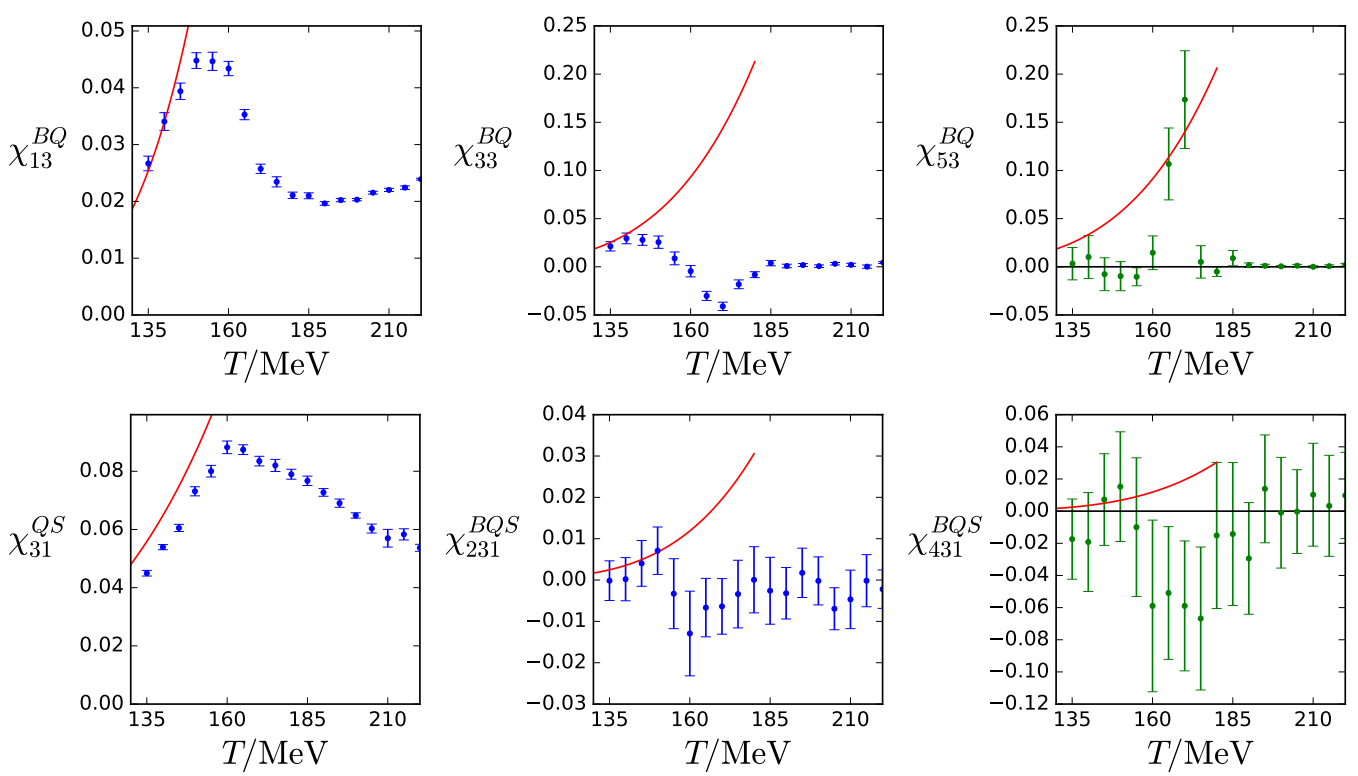

Figure 13. Results containing three electric charge derivatives on the various correlators on our $48^{3} \times 12$ lattice as functions of the temperature. Green data points denote our estimates for the high orders, these were fitted using a prior distribution. The red curves are the HRG model results. Charge correlators without baryon derivative (here $\chi_{31}^{Q S}$ ) are expected to have significant discretization errors.
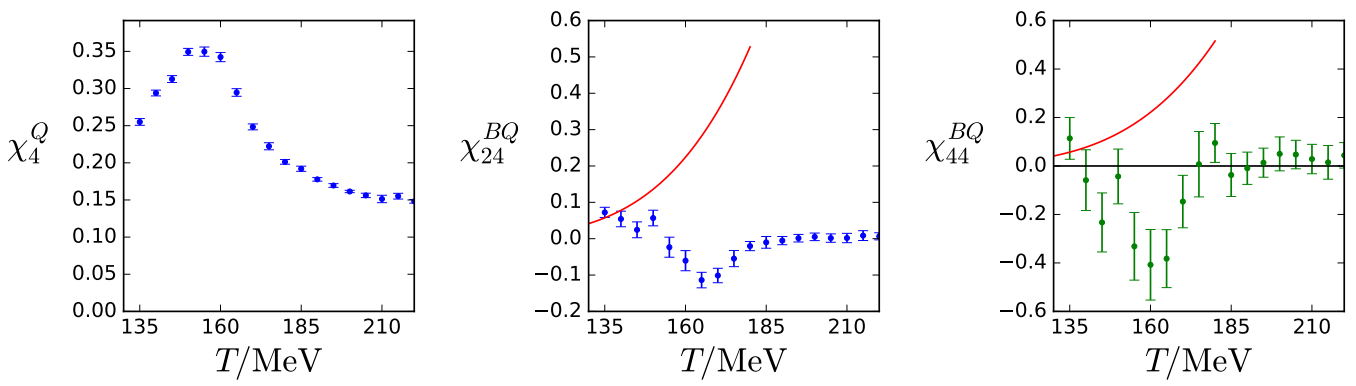

Figure 14. $\chi_{4}^{Q}, \chi_{24}^{B Q}$ and estimate for $\chi_{44}^{B Q}$ as functions of the temperature. The quantity $\chi_{4}^{Q}$ has severe cut-off effects on this lattice [28]. The red curves are the HRG model results. 


\section{B Statistics and lattice details}

In table 1 we give the number of analyzed configurations per ensemble. The simulation parameters and the details of the analysis are given in ref. [28].

The determination of the $\mu$ derivatives follows the lines of refs. [7, 28]. We calculate four quantities per configuration and per quark mass

$$
\begin{aligned}
A_{j}= & \frac{d}{d \mu_{j}} \log \left(\operatorname{det} M_{j}\right)^{1 / 4}=\frac{1}{4} \operatorname{tr} M_{j}^{-1} M_{j}^{\prime} \\
B_{j}= & \frac{d^{2}}{\left(d \mu_{j}\right)^{2}} \log \left(\operatorname{det} M_{j}\right)^{1 / 4}=\frac{1}{4} \operatorname{tr}\left(M_{j}^{\prime \prime} M_{j}^{-1}-M_{j}^{\prime} M_{j}^{-1} M_{j}^{\prime} M_{j}^{-1}\right) \\
C_{j}= & \frac{d^{3}}{\left(d \mu_{j}\right)^{3}} \log \left(\operatorname{det} M_{j}\right)^{1 / 4}=\frac{1}{4} \operatorname{tr}\left(M_{j}^{\prime} M_{j}^{-1}-3 M_{j}^{\prime \prime} M_{j}^{-1} M_{j}^{\prime} M_{j}^{-1}\right. \\
& \left.+2 M_{j}^{\prime} M_{j}^{-1} M_{j}^{\prime} M_{j}^{-1} M_{j}^{\prime} M_{j}^{-1}\right) \\
D_{j}= & \frac{d^{4}}{\left(d \mu_{j}\right)^{4}} \log \left(\operatorname{det} M_{j}\right)^{1 / 4}=\frac{1}{4} \operatorname{tr}\left(M_{j}^{\prime \prime} M_{j}^{-1}-4 M_{j}^{\prime} M_{j}^{-1} M_{j}^{\prime} M_{j}^{-1}-3 M_{j}^{\prime \prime} M_{j}^{-1} M_{j}^{\prime \prime} M_{j}^{-1}\right. \\
& +12 M_{j}^{\prime \prime} M_{j}^{-1} M_{j}^{\prime} M_{j}^{-1} M_{j}^{\prime} M_{j}^{-1} \\
& \left.-6 M_{j}^{\prime} M_{j}^{-1} M_{j}^{\prime} M_{j}^{-1} M_{j}^{\prime} M_{j}^{-1} M_{j}^{\prime} M_{j}^{-1}\right)
\end{aligned}
$$

Here $M_{j}$ is the fermion matrix corresponding to the $j$-th quark mass in the system. $M^{\prime}$ and $M^{\prime \prime}$ indicate the first and higher order derivatives with respect to the quark chemical potential. For this simple staggered action higher order derivatives are equal to lower order ones, $M^{\prime \prime \prime}=M^{\prime}$ and $M^{\prime \prime \prime \prime}=M^{\prime \prime}$ by construction. These traces are calculated using the standard stochastic method, by calculating the effect of the matrices on random sources. At finite (imaginary) chemical potentials we used $4 \times 256$ Gaussian random sources for the light quarks and $4 \times 128$ sources for the strange quarks. The analysis was accelerated by calculating 256 eigenvectors of the Dirac operator first. These eigenvectors were then fed into an Eig-CG algorithm.

Using the isospin symmetry $\left(m_{u}=m_{d}\right)$, the $A B C D$ traces can be used to calculate the $\chi^{u d s}$ derivatives with the following formulas:

$$
\begin{aligned}
& \chi_{200}^{u d s}=+\left\langle B_{u}\right\rangle+\left\langle A_{u}^{2}\right\rangle-\left\langle A_{u}\right\rangle^{2} \\
& \chi_{110}^{u d s}=+\left\langle A_{u}^{2}\right\rangle-\left\langle A_{u}\right\rangle^{2} \\
& \chi_{101}^{u d s}=+\left\langle A_{u} A_{s}\right\rangle-\left\langle A_{s}\right\rangle\left\langle A_{u}\right\rangle \\
& \chi_{300}^{u d s}=+\left\langle C_{u}\right\rangle+3\left\langle A_{u} B_{u}\right\rangle+\left\langle A_{u}^{3}\right\rangle-3\left\langle B_{u}\right\rangle\left\langle A_{u}\right\rangle-3\left\langle A_{u}\right\rangle\left\langle A_{u}^{2}\right\rangle+2\left\langle A_{u}\right\rangle^{3} \\
& \chi_{210}^{u d s}=+\left\langle A_{u} B_{u}\right\rangle+\left\langle A_{u}^{3}\right\rangle-\left\langle B_{u}\right\rangle\left\langle A_{u}\right\rangle-3\left\langle A_{u}\right\rangle\left\langle A_{u}^{3}\right\rangle+2\left\langle A_{u}\right\rangle^{3} \\
& \chi_{120}^{u d s}=+\left\langle A_{u} B_{u}\right\rangle+\left\langle A_{u}^{3}\right\rangle-\left\langle B_{u}\right\rangle\left\langle A_{u}\right\rangle-3\left\langle A_{u}\right\rangle\left\langle A_{u}^{2}\right\rangle+2\left\langle A_{u}\right\rangle^{3} \\
& \chi_{111}^{u d s}=+\left\langle A_{u} A_{u} A_{s}\right\rangle-\left\langle A_{s}\right\rangle\left\langle A_{u}^{2}\right\rangle-2\left\langle A_{u}\right\rangle\left\langle A_{u} A_{s}\right\rangle+2\left\langle A_{s}\right\rangle\left\langle A_{u}\right\rangle^{2}
\end{aligned}
$$




$$
\begin{aligned}
\chi_{400}^{u d s}= & +\left\langle D_{u}\right\rangle+3\left\langle B_{u} B_{u}\right\rangle+4\left\langle A_{u} C_{u}\right\rangle+6\left\langle A_{u}^{2} B_{u}\right\rangle+\left\langle A_{u}^{4}\right\rangle \\
& -4\left\langle C_{u}\right\rangle\left\langle A_{u}\right\rangle-3\left\langle B_{u}\right\rangle^{2}-6\left\langle B_{u}\right\rangle\left\langle A_{u}^{2}\right\rangle-12\left\langle A_{u}\right\rangle\left\langle A_{u} B_{u}\right\rangle \\
& -4\left\langle A_{u}\right\rangle\left\langle A_{u}^{3}\right\rangle-3\left\langle A_{u} A_{u}\right\rangle\left\langle A_{u}^{2}\right\rangle+12\left\langle B_{u}\right\rangle\left\langle A_{u}\right\rangle^{2} \\
& \left.+12\left\langle A_{u}\right\rangle^{2}\right\rangle\left\langle A_{u}^{2}\right\rangle-6\left\langle A_{u}\right\rangle^{4} \\
\chi_{310}^{u d s}= & +\left\langle A_{u} C_{u}\right\rangle+3\left\langle A_{u}^{2} B_{u}\right\rangle+\left\langle A_{u}^{4}\right\rangle-\left\langle C_{u}\right\rangle\left\langle A_{u}\right\rangle-3\left\langle B_{u}\right\rangle\left\langle A_{u}^{2}\right\rangle \\
& -6\left\langle A_{u}\right\rangle\left\langle A_{u} B_{u}\right\rangle-4\left\langle A_{u}\right\rangle\left\langle A_{u}^{3}\right\rangle-3\left\langle A_{u}^{2}\right\rangle\left\langle A_{u}^{2}\right\rangle \\
& +6\left\langle B_{u}\right\rangle\left\langle A_{u}\right\rangle^{2}+12\left\langle A_{u}\right\rangle\left\langle A_{u}\right\rangle\left\langle A_{u}^{2}\right\rangle-6\left\langle A_{u}\right\rangle^{4} \\
\chi_{220}^{u d s}= & +\left\langle B_{u}^{2}\right\rangle+2\left\langle A_{u}^{2} B_{u}\right\rangle+\left\langle A_{u}^{4}\right\rangle-\left\langle B_{u}\right\rangle^{2}-2\left\langle B_{u}\right\rangle\left\langle A_{u}^{2}\right\rangle \\
& -4\left\langle A_{u}\right\rangle\left\langle A_{u} B_{u}\right\rangle-4\left\langle A_{u}\right\rangle\left\langle A_{u}^{3}\right\rangle-3\left\langle A_{u}^{2}\right\rangle\left\langle A_{u}^{2}\right\rangle \\
& +4\left\langle B_{u}\right\rangle\left\langle A_{u}\right\rangle\left\langle A_{u}\right\rangle+12\left\langle A_{u}\right\rangle\left\langle A_{u}\right\rangle\left\langle A_{u}^{2}\right\rangle-6\left\langle A_{u}\right\rangle^{4} \\
\chi_{211}^{u d s}= & +\left\langle A_{u} B_{u} A_{s}\right\rangle+\left\langle A_{u}^{3} A_{s}\right\rangle-\left\langle A_{s}\right\rangle\left\langle A_{u} B_{u}\right\rangle-\left\langle A_{s}\right\rangle\left\langle A_{u}^{3}\right\rangle-\left\langle B_{u}\right\rangle\left\langle A_{u} A_{s}\right\rangle-\left\langle B_{u} A_{s}\right\rangle\left\langle A_{u}\right\rangle \\
& -3\left\langle A_{u}\right\rangle\left\langle A_{u}^{2} A_{s}\right\rangle-3\left\langle A_{u} A_{s}\right\rangle\left\langle A_{u}^{2}\right\rangle+2\left\langle A_{s}\right\rangle\left\langle B_{u}\right\rangle\left\langle A_{u}\right\rangle+6\left\langle A_{s}\right\rangle\left\langle A_{u}\right\rangle\left\langle A_{u}^{2}\right\rangle \\
& +6\left\langle A_{u}\right\rangle^{2}\left\langle A_{u} A_{s}\right\rangle-6\left\langle A_{s}\right\rangle\left\langle A_{u}\right\rangle^{3}
\end{aligned}
$$

If the listed products of the $A, B, C, D$ traces are calculated as products of the stochastic estimators, a bias could be introduced. Thus, in products different random vectors have to be used in each factor. Alternatively, the expectation value of the bias has to be subtracted. The last step is to express the derivatives in terms of $\mu_{B}, \mu_{Q}$ and $\mu_{S}$ in eq. (2.2) using eqs. (2.1), which is a straightforward exercise.

\section{Data tables}

For the reproducibility of this work we tabulate the raw data at two temperatures of this study in tables $3-6$. We have chosen one temperature below deconfinement $(140 \mathrm{MeV})$ and one above $(170 \mathrm{MeV})$, near the peak of the higher order baryon fluctuations.

For $T=140 \mathrm{MeV}$ we used the parameters: $\beta=3.7420, a m_{u d}=0.00185777, a m_{s}=$ 0.0519023 and $a m_{c}=0.615042$. For $T=170 \mathrm{MeV}$ we had $\beta=3.8236, a m_{u d}=0.00151761$, $a m_{s}=0.0420951$ and $a m_{c}=0.498827$. For a complete list of the simulation parameters see ref. [28]. In all cases we used the $\rho=0.125$ smearing parameters in four levels of stout smearing in the fat links of the standard staggered action. For the gluon fields we employed the tree-level improved Symanzik action.

In table 2 we illustrate the correlations between the mean baryon, electric charge and strangeness. Black dots means $100 \%$ correlation, red dot stands for perfect anti-correlation. The strong correlations can be understood by the relation

$$
\chi_{1}^{B}-2 \chi_{1}^{Q}+\chi_{1}^{S}=0
$$

which is exactly satisfied by our data. This relation follows from the isospin symmetric setting of our simulations with $m_{u}=m_{d}$ and $\mu_{u}=\mu_{d}$ for the $u$ and $d$ quarks. 


\begin{tabular}{|c|c|c|c|c|c|c|c|c|}
\hline$T[\mathrm{MeV}]$ & $\hat{\mu}_{B}^{I}=0$ & $\hat{\mu}_{B}^{I}=0.4$ & $\hat{\mu}_{B}^{I}=0.8$ & $\hat{\mu}_{B}^{I}=1.2$ & $\hat{\mu}_{B}^{I}=1.6$ & $\hat{\mu}_{B}^{I}=2.0$ & $\hat{\mu}_{B}^{I}=2.4$ & $\hat{\mu}_{B}^{I}=2.7$ \\
\hline 135 & 17871 & 1647 & 2680 & 4377 & 2375 & 3449 & 2622 & 2008 \\
140 & 22624 & 1625 & 3583 & 2975 & 3499 & 5321 & 3129 & 3211 \\
145 & 17195 & 2439 & 5255 & 4468 & 3191 & 2846 & 4959 & 4117 \\
150 & 18429 & 2048 & 3404 & 10115 & 6450 & 5665 & 3211 & 3254 \\
155 & 17494 & 1624 & 4735 & 4938 & 3911 & 7813 & 3670 & 3485 \\
160 & 12688 & 1607 & 4459 & 4831 & 3382 & 3917 & 4831 & 4990 \\
165 & 18472 & 1935 & 4976 & 8113 & 8466 & 4984 & 5235 & 4321 \\
170 & 14417 & 1987 & 2704 & 8820 & 8053 & 8023 & 5916 & 3273 \\
175 & 12018 & 2034 & 2006 & 4748 & 3878 & 11330 & 6178 & 5583 \\
180 & 12446 & 2104 & 2089 & 5424 & 4514 & 6057 & 5910 & 4466 \\
185 & 14184 & 2151 & 2138 & 3112 & 3086 & 5934 & 7733 & 3767 \\
190 & 13741 & 1693 & 3395 & 4395 & 8140 & 10410 & 4201 & 3844 \\
195 & 15013 & 1758 & 3643 & 5334 & 8420 & 5707 & 3884 & 4003 \\
200 & 14974 & 2300 & 2262 & 5999 & 10709 & 5033 & 5496 & 4203 \\
205 & 7788 & 2126 & 2125 & 5951 & 5873 & 8294 & 3087 & 4333 \\
210 & 4014 & 1957 & 1949 & 12174 & 6649 & 3543 & 2999 & 3146 \\
215 & 2506 & 1783 & 7056 & 2268 & 2244 & 1711 & 1674 & 2090 \\
220 & 9172 & 1810 & 3548 & 4264 & 5498 & 1754 & 1717 & 2163 \\
\hline
\end{tabular}

Table 1. Statistics of our simulations on the $48^{3} \times 12$ lattice. We list the number of stored and analyzed gauge configurations. These configurations were separated by ten Rational Hybrid Monte Carlo updates.

\begin{tabular}{|c|c|c|c|c|c|c|c|}
\hline$T / \mathrm{MeV}$ & $j=1$ & $j=2$ & $j=3$ & $j=4$ & $j=5$ & $j=6$ & $j=7$ \\
\hline 140 & & & & & & & \\
\hline 170 & & & & & & & \\
\hline
\end{tabular}

Table 2. Visualization of the correlation matrix for $\chi_{1}^{B}, \chi_{1}^{Q}$ and $\chi_{1}^{S}$ for different temperatures and $\mu_{B}^{(j)}=i T \frac{j \pi}{8}$ for $j \in\{0,1,2,3,4,5,6,7\}$. Black squares mean correlation, red squares mean anti-correlation, grey and less bright red correspond to smaller correlations. 


\begin{tabular}{|c|c|c|c|c|}
\hline obs & $j=0$ & $j=1$ & $j=2$ & $j=3$ \\
\hline$\chi_{100}^{B Q S}$ & - & $0.018 \pm 0.003$ & $0.032 \pm 0.001$ & $0.044 \pm 0.001$ \\
\hline$\chi_{200}^{B Q S}$ & $0.048 \pm 0.001$ & $0.047 \pm 0.006$ & $0.030 \pm 0.002$ & $0.018 \pm 0.002$ \\
\hline$\chi_{300}^{B Q S}$ & - & $0.034 \pm 0.025$ & $0.039 \pm 0.009$ & $0.039 \pm 0.010$ \\
\hline$\chi_{400}^{B Q S}$ & $0.052 \pm 0.014$ & $-0.001 \pm 0.093$ & $-0.043 \pm 0.038$ & $-0.017 \pm 0.045$ \\
\hline$\chi_{010}^{B Q S}$ & - & $0.004 \pm 0.001$ & $0.009 \pm 0.000$ & $0.012 \pm 0.000$ \\
\hline$\chi_{110}^{B Q S}$ & $0.013 \pm 0.000$ & $0.013 \pm 0.002$ & $0.008 \pm 0.001$ & $0.005 \pm 0.001$ \\
\hline$\chi_{210}^{B Q S}$ & - & $0.007 \pm 0.007$ & $0.011 \pm 0.003$ & $0.012 \pm 0.003$ \\
\hline$\chi_{310}^{B Q S}$ & $0.014 \pm 0.005$ & $-0.021 \pm 0.048$ & $0.009 \pm 0.016$ & $0.004 \pm 0.020$ \\
\hline$\chi_{020}^{B Q S}$ & $0.244 \pm 0.001$ & $0.245 \pm 0.003$ & $0.230 \pm 0.001$ & $0.220 \pm 0.001$ \\
\hline$\chi_{120}^{B Q S}$ & - & $0.018 \pm 0.007$ & $0.026 \pm 0.002$ & $0.039 \pm 0.003$ \\
\hline$\chi_{220}^{B Q S}$ & $0.042 \pm 0.003$ & $0.034 \pm 0.021$ & $0.021 \pm 0.009$ & $0.015 \pm 0.008$ \\
\hline$\chi_{030}^{B Q S}$ & - & $0.015 \pm 0.006$ & $0.022 \pm 0.002$ & $0.035 \pm 0.002$ \\
\hline$\chi_{130}^{B Q S}$ & $0.037 \pm 0.002$ & $0.030 \pm 0.014$ & $0.026 \pm 0.007$ & $0.019 \pm 0.007$ \\
\hline$\chi_{040}^{B Q S}$ & $0.295 \pm 0.004$ & $0.300 \pm 0.030$ & $0.268 \pm 0.010$ & $0.259 \pm 0.011$ \\
\hline$\chi_{001}^{B Q S}$ & - & $-0.009 \pm 0.002$ & $-0.015 \pm 0.000$ & $-0.020 \pm 0.001$ \\
\hline$\chi_{101}^{B Q S}$ & $-0.022 \pm 0.000$ & $-0.021 \pm 0.004$ & $-0.014 \pm 0.001$ & $-0.008 \pm 0.001$ \\
\hline$\chi_{201}^{B Q S}$ & - & $-0.020 \pm 0.012$ & $-0.016 \pm 0.004$ & $-0.015 \pm 0.004$ \\
\hline$\chi_{301}^{B Q S}$ & $-0.024 \pm 0.006$ & $-0.021 \pm 0.048$ & $0.009 \pm 0.016$ & $0.004 \pm 0.020$ \\
\hline$\chi_{002}^{B Q S}$ & $0.136 \pm 0.000$ & $0.136 \pm 0.003$ & $0.125 \pm 0.001$ & $0.117 \pm 0.001$ \\
\hline$\chi_{102}^{B Q S}$ & - & $0.022 \pm 0.009$ & $0.018 \pm 0.003$ & $0.024 \pm 0.002$ \\
\hline$\chi_{202}^{B Q S}$ & $0.031 \pm 0.003$ & $0.035 \pm 0.032$ & $0.012 \pm 0.010$ & $0.000 \pm 0.011$ \\
\hline$\chi_{003}^{B Q S}$ & - & $-0.027 \pm 0.009$ & $-0.028 \pm 0.002$ & $-0.042 \pm 0.002$ \\
\hline$\chi_{103}^{B Q S}$ & $-0.050 \pm 0.002$ & $-0.051 \pm 0.027$ & $-0.027 \pm 0.008$ & $-0.003 \pm 0.007$ \\
\hline$\chi_{004}^{B Q S}$ & $0.197 \pm 0.003$ & $0.203 \pm 0.025$ & $0.162 \pm 0.008$ & $0.126 \pm 0.008$ \\
\hline$\chi_{011}^{B Q S}$ & $0.057 \pm 0.000$ & $0.057 \pm 0.001$ & $0.055 \pm 0.000$ & $0.055 \pm 0.000$ \\
\hline$\chi_{111}^{B Q S}$ & - & $0.001 \pm 0.003$ & $0.001 \pm 0.001$ & $0.004 \pm 0.001$ \\
\hline$\chi_{211}^{B Q S}$ & $0.003 \pm 0.002$ & $0.007 \pm 0.012$ & $0.011 \pm 0.004$ & $0.002 \pm 0.006$ \\
\hline$\chi_{021}^{B Q S}$ & - & $-0.005 \pm 0.004$ & $-0.008 \pm 0.001$ & $-0.012 \pm 0.001$ \\
\hline$\chi_{121}^{B Q S}$ & $-0.014 \pm 0.001$ & $-0.016 \pm 0.011$ & $-0.005 \pm 0.003$ & $-0.003 \pm 0.003$ \\
\hline$\chi_{012}^{B Q S}$ & - & $-0.003 \pm 0.002$ & $-0.005 \pm 0.001$ & $-0.009 \pm 0.001$ \\
\hline$\chi_{112}^{B Q S}$ & $-0.009 \pm 0.001$ & $-0.008 \pm 0.006$ & $-0.008 \pm 0.002$ & $-0.001 \pm 0.003$ \\
\hline$\chi_{022}^{B Q S}$ & $0.069 \pm 0.001$ & $0.076 \pm 0.010$ & $0.061 \pm 0.002$ & $0.060 \pm 0.002$ \\
\hline$\chi_{031}^{B Q S}$ & $0.054 \pm 0.001$ & $0.057 \pm 0.011$ & $0.053 \pm 0.003$ & $0.056 \pm 0.003$ \\
\hline$\chi_{013}^{B Q S}$ & $0.073 \pm 0.001$ & $0.076 \pm 0.007$ & $0.067 \pm 0.002$ & $0.062 \pm 0.002$ \\
\hline
\end{tabular}

Table 3. Raw data at $T=140 \mathrm{MeV}$ and $\mu_{B}^{(j)}=i T \frac{j \pi}{8}$ for $j \in\{0,1,2,3\}$. 


\begin{tabular}{|c|c|c|c|c|}
\hline obs & $j=4$ & $j=5$ & $j=6$ & $j=7$ \\
\hline$\chi_{100}^{B Q S}$ & $0.047 \pm 0.001$ & $0.046 \pm 0.001$ & $0.035 \pm 0.002$ & $0.018 \pm 0.001$ \\
\hline$\chi_{200}^{B Q S}$ & $0.002 \pm 0.004$ & $-0.015 \pm 0.003$ & $-0.042 \pm 0.005$ & $-0.041 \pm 0.004$ \\
\hline$\chi_{300}^{B Q S}$ & $0.022 \pm 0.016$ & $0.028 \pm 0.011$ & $0.046 \pm 0.023$ & $0.007 \pm 0.020$ \\
\hline$\chi_{400}^{B Q S}$ & $0.005 \pm 0.070$ & $-0.058 \pm 0.065$ & $-0.105 \pm 0.113$ & $-0.135 \pm 0.113$ \\
\hline$\chi_{010}^{B Q S}$ & $0.013 \pm 0.000$ & $0.012 \pm 0.000$ & $0.009 \pm 0.000$ & $0.005 \pm 0.000$ \\
\hline$\chi_{110}^{B Q S}$ & $0.001 \pm 0.001$ & $-0.005 \pm 0.001$ & $-0.011 \pm 0.001$ & $-0.012 \pm 0.001$ \\
\hline$\chi_{210}^{B Q S}$ & $0.007 \pm 0.004$ & $0.006 \pm 0.003$ & $0.014 \pm 0.007$ & $0.001 \pm 0.006$ \\
\hline$\chi_{310}^{B Q S}$ & $-0.000 \pm 0.033$ & $0.027 \pm 0.026$ & $0.053 \pm 0.048$ & $0.063 \pm 0.048$ \\
\hline$\chi_{020}^{B Q S}$ & $0.204 \pm 0.001$ & $0.189 \pm 0.001$ & $0.176 \pm 0.001$ & $0.167 \pm 0.001$ \\
\hline$\chi_{120}^{B Q S}$ & $0.036 \pm 0.003$ & $0.033 \pm 0.002$ & $0.031 \pm 0.003$ & $0.012 \pm 0.003$ \\
\hline$\chi_{220}^{B Q S}$ & $0.005 \pm 0.010$ & $-0.021 \pm 0.011$ & $-0.044 \pm 0.017$ & $-0.048 \pm 0.013$ \\
\hline$\chi_{030}^{B Q S}$ & $0.033 \pm 0.002$ & $0.031 \pm 0.002$ & $0.027 \pm 0.003$ & $0.012 \pm 0.002$ \\
\hline$\chi_{130}^{B Q S}$ & $0.007 \pm 0.007$ & $-0.015 \pm 0.008$ & $-0.036 \pm 0.011$ & $-0.037 \pm 0.009$ \\
\hline$\chi_{040}^{B Q S}$ & $0.223 \pm 0.009$ & $0.181 \pm 0.007$ & $0.159 \pm 0.010$ & $0.138 \pm 0.010$ \\
\hline$\chi_{001}^{B Q S}$ & $-0.021 \pm 0.001$ & $-0.021 \pm 0.001$ & $-0.016 \pm 0.001$ & $-0.008 \pm 0.001$ \\
\hline$\chi_{101}^{B Q S}$ & $-0.000 \pm 0.002$ & $0.006 \pm 0.001$ & $0.019 \pm 0.002$ & $0.018 \pm 0.002$ \\
\hline$\chi_{201}^{B Q S}$ & $-0.007 \pm 0.008$ & $-0.015 \pm 0.005$ & $-0.018 \pm 0.010$ & $-0.006 \pm 0.009$ \\
\hline$\chi_{301}^{B Q S}$ & $-0.000 \pm 0.033$ & $0.027 \pm 0.026$ & $0.053 \pm 0.048$ & $0.063 \pm 0.048$ \\
\hline$\chi_{002}^{B Q S}$ & $0.105 \pm 0.001$ & $0.096 \pm 0.001$ & $0.083 \pm 0.001$ & $0.080 \pm 0.001$ \\
\hline$\chi_{102}^{B Q S}$ & $0.020 \pm 0.005$ & $0.025 \pm 0.003$ & $0.022 \pm 0.005$ & $0.009 \pm 0.005$ \\
\hline $\begin{array}{l}\chi_{202}^{B Q S} \\
\end{array}$ & $-0.002 \pm 0.018$ & $-0.021 \pm 0.013$ & $-0.050 \pm 0.026$ & $-0.050 \pm 0.021$ \\
\hline$\chi_{003}^{B Q S}$ & $-0.040 \pm 0.003$ & $-0.042 \pm 0.002$ & $-0.035 \pm 0.003$ & $-0.016 \pm 0.003$ \\
\hline$\chi_{103}^{B Q S}$ & $0.004 \pm 0.012$ & $0.021 \pm 0.009$ & $0.057 \pm 0.016$ & $0.053 \pm 0.011$ \\
\hline$\chi_{004}^{B Q S}$ & $0.099 \pm 0.010$ & $0.075 \pm 0.008$ & $0.030 \pm 0.013$ & $0.024 \pm 0.009$ \\
\hline$\chi_{011}^{B Q S}$ & $0.052 \pm 0.000$ & $0.051 \pm 0.000$ & $0.051 \pm 0.001$ & $0.049 \pm 0.001$ \\
\hline$\chi_{111}^{B Q S}$ & $0.007 \pm 0.002$ & $0.005 \pm 0.001$ & $0.002 \pm 0.003$ & $0.002 \pm 0.003$ \\
\hline$\chi_{211}^{B Q S}$ & $-0.001 \pm 0.009$ & $0.003 \pm 0.008$ & $0.001 \pm 0.013$ & $0.007 \pm 0.014$ \\
\hline$\chi_{021}^{B Q S}$ & $-0.010 \pm 0.001$ & $-0.010 \pm 0.001$ & $-0.009 \pm 0.001$ & $-0.004 \pm 0.001$ \\
\hline$\chi_{121}^{B Q S}$ & $-0.000 \pm 0.004$ & $0.007 \pm 0.003$ & $0.014 \pm 0.006$ & $0.017 \pm 0.005$ \\
\hline$\chi_{012}^{B Q S}$ & $-0.010 \pm 0.001$ & $-0.009 \pm 0.001$ & $-0.006 \pm 0.001$ & $-0.003 \pm 0.001$ \\
\hline$\chi_{112}^{B Q S}$ & $0.001 \pm 0.005$ & $-0.000 \pm 0.004$ & $0.003 \pm 0.006$ & $0.002 \pm 0.006$ \\
\hline$\chi_{022}^{B Q S}$ & $0.050 \pm 0.003$ & $0.043 \pm 0.002$ & $0.040 \pm 0.003$ & $0.033 \pm 0.002$ \\
\hline$\chi_{031}^{B Q S}$ & $0.049 \pm 0.003$ & $0.050 \pm 0.003$ & $0.055 \pm 0.004$ & $0.051 \pm 0.003$ \\
\hline$\chi_{013}^{B Q S}$ & $0.051 \pm 0.003$ & $0.048 \pm 0.002$ & $0.043 \pm 0.004$ & $0.039 \pm 0.003$ \\
\hline
\end{tabular}

Table 4. Raw data at $T=140 \mathrm{MeV}$ and $\mu_{B}^{(j)}=i T \frac{j \pi}{8}$ for $j \in\{4,5,6,7\}$. 


\begin{tabular}{|c|c|c|c|c|}
\hline obs & $j=0$ & $j=1$ & $j=2$ & $j=3$ \\
\hline$\chi_{100}^{B Q S}$ & - & $0.067 \pm 0.001$ & $0.130 \pm 0.002$ & $0.181 \pm 0.001$ \\
\hline$\chi_{200}^{B Q S}$ & $0.173 \pm 0.001$ & $0.163 \pm 0.003$ & $0.147 \pm 0.004$ & $0.112 \pm 0.002$ \\
\hline$\chi_{300}^{B Q S}$ & - & $0.029 \pm 0.011$ & $0.079 \pm 0.011$ & $0.113 \pm 0.009$ \\
\hline$\chi_{400}^{B Q S}$ & $0.069 \pm 0.011$ & $0.045 \pm 0.045$ & $0.096 \pm 0.045$ & $0.108 \pm 0.039$ \\
\hline$\chi_{010}^{B Q S}$ & - & $0.011 \pm 0.000$ & $0.022 \pm 0.000$ & $0.031 \pm 0.000$ \\
\hline$\chi_{110}^{B Q S}$ & $0.029 \pm 0.000$ & $0.027 \pm 0.001$ & $0.026 \pm 0.001$ & $0.022 \pm 0.000$ \\
\hline$\chi_{210}^{B Q S}$ & - & $0.004 \pm 0.002$ & $0.012 \pm 0.002$ & $0.015 \pm 0.002$ \\
\hline$\chi_{310}^{B Q S}$ & $0.007 \pm 0.002$ & $-0.058 \pm 0.031$ & $-0.054 \pm 0.030$ & $-0.055 \pm 0.025$ \\
\hline$\chi_{020}^{B Q S}$ & $0.474 \pm 0.001$ & $0.471 \pm 0.002$ & $0.456 \pm 0.002$ & $0.434 \pm 0.001$ \\
\hline$\chi_{120}^{B Q S}$ & - & $0.020 \pm 0.003$ & $0.048 \pm 0.003$ & $0.068 \pm 0.002$ \\
\hline$\chi_{220}^{B Q S}$ & $0.049 \pm 0.002$ & $0.052 \pm 0.009$ & $0.061 \pm 0.008$ & $0.071 \pm 0.007$ \\
\hline$\chi_{030}^{B Q S}$ & - & $0.011 \pm 0.002$ & $0.027 \pm 0.002$ & $0.038 \pm 0.001$ \\
\hline$\chi_{130}^{B Q S}$ & $0.025 \pm 0.001$ & $0.029 \pm 0.005$ & $0.037 \pm 0.005$ & $0.045 \pm 0.004$ \\
\hline$\chi_{040}^{B Q S}$ & $0.249 \pm 0.004$ & $0.260 \pm 0.015$ & $0.286 \pm 0.015$ & $0.302 \pm 0.008$ \\
\hline$\chi_{001}^{B Q S}$ & - & $-0.045 \pm 0.001$ & $-0.086 \pm 0.001$ & $-0.119 \pm 0.001$ \\
\hline$\chi_{101}^{B Q S}$ & $-0.115 \pm 0.001$ & $-0.110 \pm 0.002$ & $-0.096 \pm 0.003$ & $-0.069 \pm 0.002$ \\
\hline$\chi_{201}^{B Q S}$ & - & $-0.020 \pm 0.008$ & $-0.055 \pm 0.008$ & $-0.082 \pm 0.006$ \\
\hline$\chi_{301}^{B Q S}$ & $-0.055 \pm 0.007$ & $-0.058 \pm 0.031$ & $-0.054 \pm 0.030$ & $-0.055 \pm 0.025$ \\
\hline$\chi_{002}^{B Q S}$ & $0.411 \pm 0.001$ & $0.405 \pm 0.003$ & $0.381 \pm 0.004$ & $0.339 \pm 0.002$ \\
\hline$\chi_{102}^{B Q S}$ & - & $0.034 \pm 0.008$ & $0.083 \pm 0.008$ & $0.121 \pm 0.005$ \\
\hline $\begin{array}{l}\chi_{202}^{B Q S} \\
\end{array}$ & $0.093 \pm 0.006$ & $0.111 \pm 0.029$ & $0.083 \pm 0.027$ & $0.077 \pm 0.020$ \\
\hline$\chi_{003}^{B Q S}$ & - & $-0.071 \pm 0.009$ & $-0.160 \pm 0.011$ & $-0.226 \pm 0.005$ \\
\hline$\chi_{103}^{B Q S}$ & $-0.195 \pm 0.006$ & $-0.214 \pm 0.030$ & $-0.166 \pm 0.028$ & $-0.142 \pm 0.020$ \\
\hline$\chi_{004}^{B Q S}$ & $0.600 \pm 0.008$ & $0.605 \pm 0.038$ & $0.551 \pm 0.034$ & $0.486 \pm 0.022$ \\
\hline$\chi_{011}^{B Q S}$ & $0.148 \pm 0.000$ & $0.148 \pm 0.001$ & $0.142 \pm 0.001$ & $0.135 \pm 0.001$ \\
\hline$\chi_{111}^{B Q S}$ & - & $0.007 \pm 0.001$ & $0.014 \pm 0.001$ & $0.019 \pm 0.001$ \\
\hline$\chi_{211}^{B Q S}$ & $0.019 \pm 0.001$ & $0.027 \pm 0.003$ & $0.014 \pm 0.004$ & $0.011 \pm 0.004$ \\
\hline$\chi_{021}^{B Q S}$ & - & $-0.013 \pm 0.002$ & $-0.029 \pm 0.002$ & $-0.042 \pm 0.001$ \\
\hline$\chi_{121}^{B Q S}$ & $-0.034 \pm 0.001$ & $-0.035 \pm 0.005$ & $-0.034 \pm 0.005$ & $-0.035 \pm 0.004$ \\
\hline$\chi_{012}^{B Q S}$ & - & $-0.018 \pm 0.001$ & $-0.038 \pm 0.002$ & $-0.053 \pm 0.001$ \\
\hline$\chi_{112}^{B Q S}$ & $-0.051 \pm 0.001$ & $-0.051 \pm 0.003$ & $-0.041 \pm 0.003$ & $-0.033 \pm 0.003$ \\
\hline$\chi_{022}^{B Q S}$ & $0.129 \pm 0.002$ & $0.129 \pm 0.008$ & $0.135 \pm 0.008$ & $0.131 \pm 0.004$ \\
\hline$\chi_{031}^{B Q S}$ & $0.083 \pm 0.002$ & $0.082 \pm 0.005$ & $0.089 \pm 0.006$ & $0.085 \pm 0.003$ \\
\hline$\chi_{013}^{B Q S}$ & $0.203 \pm 0.002$ & $0.195 \pm 0.006$ & $0.193 \pm 0.007$ & $0.172 \pm 0.003$ \\
\hline
\end{tabular}

Table 5. Raw data at $T=170 \mathrm{MeV}$ and $\mu_{B}^{(j)}=i T \frac{j \pi}{8}$ for $j \in\{0,1,2,3\}$. 


\begin{tabular}{|c|c|c|c|c|}
\hline obs & $j=4$ & $j=5$ & $j=6$ & $j=7$ \\
\hline$\chi_{100}^{B Q S}$ & $0.213 \pm 0.001$ & $0.225 \pm 0.002$ & $0.180 \pm 0.003$ & $0.109 \pm 0.005$ \\
\hline$\chi_{200}^{B Q S}$ & $0.055 \pm 0.004$ & $-0.034 \pm 0.008$ & $-0.143 \pm 0.012$ & $-0.245 \pm 0.021$ \\
\hline$\chi_{300}^{B Q S}$ & $0.180 \pm 0.016$ & $0.342 \pm 0.057$ & $0.334 \pm 0.065$ & $0.174 \pm 0.168$ \\
\hline$\chi_{400}^{B Q S}$ & $0.167 \pm 0.093$ & $0.588 \pm 0.408$ & $-0.698 \pm 0.415$ & $0.223 \pm 1.184$ \\
\hline$\chi_{010}^{B Q S}$ & $0.038 \pm 0.000$ & $0.041 \pm 0.000$ & $0.034 \pm 0.001$ & $0.021 \pm 0.001$ \\
\hline$\chi_{110}^{B Q S}$ & $0.013 \pm 0.001$ & $-0.003 \pm 0.001$ & $-0.026 \pm 0.002$ & $-0.046 \pm 0.004$ \\
\hline$\chi_{210}^{B Q S}$ & $0.029 \pm 0.003$ & $0.065 \pm 0.011$ & $0.071 \pm 0.012$ & $0.036 \pm 0.032$ \\
\hline$\chi_{310}^{B Q S}$ & $-0.101 \pm 0.064$ & $-0.329 \pm 0.244$ & $0.478 \pm 0.268$ & $-0.135 \pm 0.739$ \\
\hline$\chi_{020}^{B Q S}$ & $0.399 \pm 0.001$ & $0.359 \pm 0.001$ & $0.298 \pm 0.001$ & $0.257 \pm 0.002$ \\
\hline$\chi_{120}^{B Q S}$ & $0.099 \pm 0.003$ & $0.135 \pm 0.006$ & $0.139 \pm 0.006$ & $0.094 \pm 0.008$ \\
\hline$\chi_{220}^{B Q S}$ & $0.080 \pm 0.013$ & $0.119 \pm 0.039$ & $-0.045 \pm 0.034$ & $-0.174 \pm 0.066$ \\
\hline$\chi_{030}^{B Q S}$ & $0.058 \pm 0.001$ & $0.080 \pm 0.003$ & $0.085 \pm 0.003$ & $0.059 \pm 0.004$ \\
\hline$\chi_{130}^{B Q S}$ & $0.050 \pm 0.006$ & $0.075 \pm 0.017$ & $-0.012 \pm 0.015$ & $-0.121 \pm 0.033$ \\
\hline$\chi_{040}^{B Q S}$ & $0.326 \pm 0.009$ & $0.354 \pm 0.012$ & $0.290 \pm 0.010$ & $0.184 \pm 0.013$ \\
\hline$\chi_{001}^{B Q S}$ & $-0.137 \pm 0.001$ & $-0.143 \pm 0.001$ & $-0.112 \pm 0.002$ & $-0.067 \pm 0.003$ \\
\hline$\chi_{101}^{B Q S}$ & $-0.030 \pm 0.003$ & $0.028 \pm 0.006$ & $0.091 \pm 0.008$ & $0.153 \pm 0.013$ \\
\hline$\chi_{201}^{B Q S}$ & $-0.123 \pm 0.011$ & $-0.212 \pm 0.036$ & $-0.191 \pm 0.043$ & $-0.102 \pm 0.104$ \\
\hline$\chi_{301}^{B Q S}$ & $-0.101 \pm 0.064$ & $-0.329 \pm 0.244$ & $0.478 \pm 0.268$ & $-0.135 \pm 0.739$ \\
\hline$\chi_{002}^{B Q S}$ & $0.282 \pm 0.003$ & $0.214 \pm 0.004$ & $0.134 \pm 0.005$ & $0.070 \pm 0.009$ \\
\hline$\chi_{102}^{B Q S}$ & $0.165 \pm 0.009$ & $0.228 \pm 0.026$ & $0.197 \pm 0.029$ & $0.118 \pm 0.064$ \\
\hline$\chi_{202}^{B Q S}$ & $0.104 \pm 0.050$ & $0.213 \pm 0.161$ & $-0.362 \pm 0.178$ & $-0.043 \pm 0.464$ \\
\hline$\chi_{003}^{B Q S}$ & $-0.287 \pm 0.009$ & $-0.340 \pm 0.020$ & $-0.286 \pm 0.020$ & $-0.179 \pm 0.040$ \\
\hline$\chi_{103}^{B Q S}$ & $-0.131 \pm 0.045$ & $-0.126 \pm 0.113$ & $0.352 \pm 0.123$ & $0.258 \pm 0.299$ \\
\hline$\chi_{004}^{B Q S}$ & $0.414 \pm 0.045$ & $0.268 \pm 0.084$ & $-0.212 \pm 0.093$ & $-0.302 \pm 0.206$ \\
\hline$\chi_{011}^{B Q S}$ & $0.126 \pm 0.001$ & $0.121 \pm 0.001$ & $0.113 \pm 0.002$ & $0.112 \pm 0.002$ \\
\hline$\chi_{111}^{B Q S}$ & $0.021 \pm 0.002$ & $0.008 \pm 0.006$ & $0.003 \pm 0.008$ & $0.008 \pm 0.020$ \\
\hline$\chi_{211}^{B Q S}$ & $0.001 \pm 0.008$ & $-0.058 \pm 0.043$ & $0.058 \pm 0.049$ & $-0.089 \pm 0.142$ \\
\hline$\chi_{021}^{B Q S}$ & $-0.057 \pm 0.002$ & $-0.074 \pm 0.003$ & $-0.071 \pm 0.004$ & $-0.047 \pm 0.004$ \\
\hline$\chi_{121}^{B Q S}$ & $-0.039 \pm 0.008$ & $-0.049 \pm 0.021$ & $0.038 \pm 0.020$ & $0.084 \pm 0.036$ \\
\hline$\chi_{012}^{B Q S}$ & $-0.061 \pm 0.002$ & $-0.056 \pm 0.004$ & $-0.044 \pm 0.005$ & $-0.030 \pm 0.013$ \\
\hline$\chi_{112}^{B Q S}$ & $-0.014 \pm 0.005$ & $0.044 \pm 0.025$ & $-0.005 \pm 0.033$ & $0.107 \pm 0.090$ \\
\hline$\chi_{022}^{B Q S}$ & $0.133 \pm 0.006$ & $0.129 \pm 0.013$ & $0.069 \pm 0.011$ & $0.039 \pm 0.020$ \\
\hline$\chi_{031}^{B Q S}$ & $0.083 \pm 0.004$ & $0.069 \pm 0.008$ & $0.100 \pm 0.010$ & $0.133 \pm 0.019$ \\
\hline$\chi_{013}^{B Q S}$ & $0.142 \pm 0.005$ & $0.071 \pm 0.016$ & $0.070 \pm 0.022$ & $-0.022 \pm 0.056$ \\
\hline
\end{tabular}

Table 6. Raw data at $T=170 \mathrm{MeV}$ and $\mu_{B}^{(j)}=i T \frac{j \pi}{8}$ for $j \in\{4,5,6,7\}$. 
Open Access. This article is distributed under the terms of the Creative Commons Attribution License (CC-BY 4.0), which permits any use, distribution and reproduction in any medium, provided the original author(s) and source are credited.

\section{References}

[1] Y. Aoki, G. Endrodi, Z. Fodor, S.D. Katz and K.K. Szabo, The Order of the quantum chromodynamics transition predicted by the standard model of particle physics, Nature $\mathbf{4 4 3}$ (2006) 675 [hep-lat/0611014] [INSPIRE].

[2] Y. Aoki, Z. Fodor, S.D. Katz and K.K. Szabo, The QCD transition temperature: Results with physical masses in the continuum limit, Phys. Lett. B 643 (2006) 46 [hep-lat/0609068] [INSPIRE].

[3] Y. Aoki et al., The QCD transition temperature: results with physical masses in the continuum limit II., JHEP 06 (2009) 088 [arXiv:0903.4155] [INSPIRE].

[4] Wuppertal-BudApest collaboration, S. Borsányi et al., Is there still any $T_{c}$ mystery in lattice QCD? Results with physical masses in the continuum limit III, JHEP 09 (2010) 073 [arXiv: 1005.3508] [INSPIRE].

[5] T. Bhattacharya et al., QCD Phase Transition with Chiral Quarks and Physical Quark Masses, Phys. Rev. Lett. 113 (2014) 082001 [arXiv:1402.5175] [INSPIRE].

[6] A. Bazavov et al., The chiral and deconfinement aspects of the QCD transition, Phys. Rev. D 85 (2012) 054503 [arXiv: 1111.1710] [INSPIRE].

[7] C.R. Allton et al., The QCD thermal phase transition in the presence of a small chemical potential, Phys. Rev. D 66 (2002) 074507 [hep-lat/0204010] [INSPIRE].

[8] C.R. Allton et al., Thermodynamics of two flavor QCD to sixth order in quark chemical potential, Phys. Rev. D 71 (2005) 054508 [hep-lat/0501030] [INSPIRE].

[9] R.V. Gavai and S. Gupta, QCD at finite chemical potential with six time slices, Phys. Rev. D 78 (2008) 114503 [arXiv:0806.2233] [INSPIRE].

[10] MILC collaboration, S. Basak et al., QCD equation of state at non-zero chemical potential, PoS (LATTICE2008) 171 (2008) [arXiv:0910.0276] [INSPIRE].

[11] O. Kaczmarek et al., Phase boundary for the chiral transition in (2+1)-flavor QCD at small values of the chemical potential, Phys. Rev. D 83 (2011) 014504 [arXiv:1011.3130] [INSPIRE].

[12] Z. Fodor and S.D. Katz, A New method to study lattice QCD at finite temperature and chemical potential, Phys. Lett. B 534 (2002) 87 [hep-lat/0104001] [INSPIRE].

[13] P. de Forcrand and O. Philipsen, The QCD phase diagram for small densities from imaginary chemical potential, Nucl. Phys. B 642 (2002) 290 [hep-lat/0205016] [InSPIRE].

[14] M. D'Elia and M.-P. Lombardo, Finite density QCD via imaginary chemical potential, Phys. Rev. D 67 (2003) 014505 [hep-lat/0209146] [INSPIRE].

[15] Z. Fodor and S.D. Katz, Lattice determination of the critical point of QCD at finite $T$ and mu, JHEP 03 (2002) 014 [hep-lat/0106002] [INSPIRE].

[16] Z. Fodor and S.D. Katz, Critical point of QCD at finite $T$ and $m u$, lattice results for physical quark masses, JHEP 04 (2004) 050 [hep-lat/0402006] [INSPIRE]. 
[17] C. Bonati, M. D'Elia, F. Negro, F. Sanfilippo and K. Zambello, Curvature of the pseudocritical line in QCD: Taylor expansion matches analytic continuation, Phys. Rev. D 98 (2018) 054510 [arXiv: 1805.02960] [INSPIRE].

[18] F. Karsch, Determination of Freeze-out Conditions from Lattice QCD Calculations, Central Eur. J. Phys. 10 (2012) 1234 [arXiv:1202.4173] [InSPIRE].

[19] A. Bazavov et al., Freeze-out Conditions in Heavy Ion Collisions from QCD Thermodynamics, Phys. Rev. Lett. 109 (2012) 192302 [arXiv:1208.1220] [INSPIRE].

[20] S. Borsányi, Z. Fodor, S.D. Katz, S. Krieg, C. Ratti and K.K. Szabo, Freeze-out parameters: lattice meets experiment, Phys. Rev. Lett. 111 (2013) 062005 [arXiv:1305.5161] [INSPIRE].

[21] S. Borsányi, Z. Fodor, S.D. Katz, S. Krieg, C. Ratti and K.K. Szabo, Freeze-out parameters from electric charge and baryon number fluctuations: is there consistency?, Phys. Rev. Lett. 113 (2014) 052301 [arXiv: 1403.4576] [INSPIRE].

[22] C. Ratti, Lattice QCD and heavy ion collisions: a review of recent progress, Rept. Prog. Phys. 81 (2018) 084301 [arXiv: 1804.07810] [INSPIRE].

[23] НотQCD collaboration, A. Bazavov et al., Skewness and kurtosis of net baryon-number distributions at small values of the baryon chemical potential, Phys. Rev. D 96 (2017) 074510 [arXiv: 1708.04897] [INSPIRE].

[24] M.A. Stephanov, K. Rajagopal and E.V. Shuryak, Event-by-event fluctuations in heavy ion collisions and the QCD critical point, Phys. Rev. D 60 (1999) 114028 [hep-ph/9903292] [INSPIRE].

[25] M. Cheng et al., The QCD equation of state with almost physical quark masses, Phys. Rev. D 77 (2008) 014511 [arXiv:0710.0354] [INSPIRE].

[26] J.N. Guenther et al., The QCD equation of state at finite density from analytical continuation, Nucl. Phys. A 967 (2017) 720 [arXiv:1607.02493] [INSPIRE].

[27] M. D'Elia, G. Gagliardi and F. Sanfilippo, Higher order quark number fluctuations via imaginary chemical potentials in $N_{f}=2+1$ QCD, Phys. Rev. D 95 (2017) 094503 [arXiv: 1611.08285] [INSPIRE].

[28] R. Bellwied et al., Fluctuations and correlations in high temperature QCD, Phys. Rev. D 92 (2015) 114505 [arXiv: 1507.04627] [INSPIRE].

[29] A. Roberge and N. Weiss, Gauge Theories With Imaginary Chemical Potential and the Phases of QCD, Nucl. Phys. B 275 (1986) 734 [InSPIRE].

[30] V. Vovchenko, A. Pasztor, Z. Fodor, S.D. Katz and H. Stoecker, Repulsive baryonic interactions and lattice QCD observables at imaginary chemical potential, Phys. Lett. B 775 (2017) 71 [arXiv:1708.02852] [INSPIRE].

[31] J.I. Kapusta and C. Gale, Finite-Temperature Field Theory, second edition, Cambridge University Press (2006).

[32] A. Vuorinen, Quark number susceptibilities of hot QCD up to $g^{* *} 6 \ln g$, Phys. Rev. D 67 (2003) 074032 [hep-ph/0212283] [INSPIRE].

[33] O. Philipsen and C. Pinke, Nature of the Roberge-Weiss transition in $N_{f}=2 Q C D$ with Wilson fermions, Phys. Rev. D 89 (2014) 094504 [arXiv: 1402.0838] [INSPIRE].

[34] C. Czaban, F. Cuteri, O. Philipsen, C. Pinke and A. Sciarra, Roberge-Weiss transition in $N_{f}=2$ QCD with Wilson fermions and $N_{\tau}=6$, Phys. Rev. D 93 (2016) 054507 [arXiv: 1512.07180] [INSPIRE]. 
[35] C. Bonati, M. D'Elia, M. Mariti, M. Mesiti, F. Negro and F. Sanfilippo, Roberge-Weiss endpoint at the physical point of $N_{f}=2+1$ QCD, Phys. Rev. D 93 (2016) 074504 [arXiv: 1602.01426] [INSPIRE].

[36] C. Bonati, M. D'Elia, M. Mariti, M. Mesiti, F. Negro and F. Sanfilippo, Curvature of the chiral pseudocritical line in QCD: Continuum extrapolated results, Phys. Rev. D 92 (2015) 054503 [arXiv: 1507.03571] [INSPIRE].

[37] R. Bellwied et al., The QCD phase diagram from analytic continuation, Phys. Lett. B 751 (2015) 559 [arXiv: 1507.07510] [inSPIRE].

[38] P. Cea, L. Cosmai and A. Papa, Critical line of 2+1 flavor QCD: Toward the continuum limit, Phys. Rev. D 93 (2016) 014507 [arXiv: 1508.07599] [INSPIRE].

[39] A. Bazavov et al., The QCD Equation of State to $\mathcal{O}\left(\mu_{B}^{6}\right)$ from Lattice $Q C D$, Phys. Rev. D 95 (2017) 054504 [arXiv: 1701.04325] [INSPIRE].

[40] C. McNeile, C.T.H. Davies, E. Follana, K. Hornbostel and G.P. Lepage, High-Precision c and $b$ Masses and QCD Coupling from Current-Current Correlators in Lattice and Continuum QCD, Phys. Rev. D 82 (2010) 034512 [arXiv: 1004.4285] [INSPIRE].

[41] N. Haque, J.O. Andersen, M.G. Mustafa, M. Strickland and N. Su, Three-loop pressure and susceptibility at finite temperature and density from hard-thermal-loop perturbation theory, Phys. Rev. D 89 (2014) 061701 [arXiv: 1309.3968] [INSPIRE].

[42] N. Haque, A. Bandyopadhyay, J.O. Andersen, M.G. Mustafa, M. Strickland and N. Su, Three-loop HTLpt thermodynamics at finite temperature and chemical potential, JHEP 05 (2014) 027 [arXiv:1402.6907] [INSPIRE].

[43] H.T. Ding, S. Mukherjee, H. Ohno, P. Petreczky and H.P. Schadler, Diagonal and off-diagonal quark number susceptibilities at high temperatures, Phys. Rev. D 92 (2015) 074043 [arXiv: 1507.06637] [INSPIRE].

[44] H. Akaike, A New Look at the Statistical Model Identification, IEEE Trans. Automat. Contr. 19 (1974) 716.

[45] S. Dürr et al., Ab-Initio Determination of Light Hadron Masses, Science 322 (2008) 1224 [arXiv:0906.3599] [INSPIRE].

[46] STAR collaboration, X. Luo, Energy Dependence of Moments of Net-Proton and Net-Charge Multiplicity Distributions at STAR, PoS (CPOD2014) 019 (2015) [arXiv:1503. 02558] [INSPIRE].

[47] STAR collaboration, J. Thäder, Higher Moments of Net-Particle Multiplicity Distributions, Nucl. Phys. A 956 (2016) 320 [arXiv:1601.00951] [INSPIRE].

[48] A. Andronic, P. Braun-Munzinger and J. Stachel, Hadron production in central nucleus-nucleus collisions at chemical freeze-out, Nucl. Phys. A 772 (2006) 167 [nucl-th/0511071] [INSPIRE].

[49] P. Alba et al., Constraining the hadronic spectrum through QCD thermodynamics on the lattice, Phys. Rev. D 96 (2017) 034517 [arXiv:1702.01113] [InSPIRE].

[50] Jülich Supercomputing Centre, JUQUEEN: IBM Blue Gene/Q Supercomputer System at the Jülich Supercomputing Centre, JLSRF 1 (2015) A1. 